\title{
Transcriptome dynamics of hippocampal neurogenesis in macaques across the lifespan and aged humans
}

\section{Xiaoqun Wang ( $\nabla$ xiaoqunwang@ibp.ac.cn )}

Institute of Biophysics, Chinese Academy of Sciences https://orcid.org/0000-0003-3440-2617

\section{Wei Wang}

Institute of Biophysics, Chinese Academy of Sciences

\section{Mengdi Wang}

Institute of Biophysics, Chinese Academy of Sciences

\section{Meng Yang}

State Key Laboratory of Brain and Cognitive Science, CAS Center for Excellence in Brain Science and Intelligence Technology (Shanghai), Institute of Biophysics, Chinese Academy of Sciences, Beijing

\section{Bo Zeng}

Institute of Biophysics, Chinese Academy of Sciences

\section{Wenying Qiu}

Peking Union Medical College

\section{Qiang Ma}

Institute of Biophysics, Chinese Academy of Sciences

\section{Xiaoxi Jing}

Beijing Normal University

\section{Qianqian Zhang}

University of Chinese Academy of Sciences

\section{Bosong Wang}

Beijing Normal University

\section{Chonghai Yin}

Bioland Laboratory (Guangzhou Regenerative Medicine and Health Guangdong Laboratory)

Jiyao Zhang

Beijing Normal University

\section{Yuxin Ge}

Beijing Normal University

\section{Yufeng Lu}

Institute of Biophysics, Chinese Academy of Sciences

\section{Weizhi Ji}


Institute of Primate Translational Medicine, Kunming University of Science and Technology https://orcid.org/0000-0003-2550-4224

\section{Qian Wu}

Beijing Normal University https://orcid.org/0000-0002-7469-1583

\section{Chao Ma}

Chinese Academy of Medical Sciences, Peking Union Medical Collage

\section{Biological Sciences - Article}

Keywords: adult hippocampal neurogenesis, single nucleus RNA sequencing, neural stem cells

Posted Date: November 18th, 2021

DOI: https://doi.org/10.21203/rs.3.rs-1015150/v1

License: (c) (1) This work is licensed under a Creative Commons Attribution 4.0 International License. Read Full License 
2 macaques across the lifespan and aged humans

3 Wei Wang ${ }^{1,5,9}$, Mengdi Wang ${ }^{1,5,9}$, Meng Yang ${ }^{1,5,9}$, Bo Zeng ${ }^{1,5,9}$, Wenying Qiü ${ }^{2,9}$, Qiang Ma ${ }^{1,5}$,

4 Xiaoxi Jing 3 , Qianqian Zhang, ${ }^{1,5}$ Baisong Wang 3 , Chonghai Yin ${ }^{4}$, Jiyao Zhang ${ }^{3}$, Yuxin $\mathrm{Ge}^{3}$,

5 Yufeng $\mathrm{Lu}^{1,5}$, Weizhi $\mathrm{Ji}^{6}$, Qian $\mathrm{Wu}^{3,}$, Chao $\mathrm{Ma}^{2,}$, , Xiaoqun Wang ${ }^{1,4,5,6,7,8, \text { * }}$

6

$7 \quad 1$ State Key Laboratory of Brain and Cognitive Science, CAS Center for Excellence in Brain

8 Science and Intelligence Technology (Shanghai), Institute of Biophysics, Chinese Academy of

9 Sciences, Beijing 100101, China.

102 Institute of Basic Medical Sciences, Neuroscience Center, National Human Brain Bank for

11 Development and Function, Chinese Academy of Medical Sciences; Department of Human

12 Anatomy, Histology and Embryology, School of Basic Medicine, Peking Union Medical

13 College, Beijing 100005, China.

143 State Key Laboratory of Cognitive Neuroscience and Learning, IDG/McGovern Institute for

Brain Research, Beijing Normal University, Beijing, 100875, China.

4 Bioland Laboratory (Guangzhou Regenerative Medicine and Health Guangdong Laboratory), Guangzhou, 510005, China.

185 University of Chinese Academy of Sciences, Beijing 100049, China.

6 Yunnan Key Laboratory of Primate Biomedical Research, Institute of Primate Translational Medicine, Kunming University of Science and Technology, Kunming, 650500, China.

7 Institute for Stem Cell and Regeneration, CAS, Beijing 100101, China. Disorders, Capital Medical University, Beijing, 100069, China. 9 These authors contributed equally. 


\section{Abstract}

32 Whether adult hippocampal neurogenesis (AHN) persists in adult and aged humans remains 33 extensive debates ${ }^{1-8}$. Here, to provide a better understanding of AHN of primates, droplet-based 34 single nucleus RNA sequencing (snRNA-seq) is used to investigate the cellular heterogeneity 35 and molecular characteristics of the hippocampi in macaques across the lifespan and in aged 36 humans. We pinpoint the dynamics of the neurogenic lineage, including adult neural stem cells 37 (NSCs) and immature neurons, and the diversity of astrocytes and microglia. In the neurogenic 38 lineage, the regulatory continuum from adult NSCs to immature and mature granule cells is 39 investigated. We identify ETNPPL as a primate-specific NSC marker and verify STMN1 and 40 STMN2 as immature neuron markers in primates. Importantly, we also illustrate a cluster of 41 active astrocytes and microglia exhibiting proinflammatory responses in aged samples. The 42 interaction analysis implies that astrocytes are more important niche cells that provide signals 43 inducing the proliferation, quiescence and inflammation of adult NSCs at different stages and 44 thus are attributed to the decrease and variability of AHN in adult and elderly. 
The hippocampal formation is one of the main brain regions affected in neurological diseases, such as Alzheimer's disease, stress, and depression, and has attracted tremendous attention due to its physiological and clinical significance ${ }^{9,10}$. Extensive evidence demonstrates that hippocampal neurogenesis is involved in memory processing, cognitive function, and mood regulation in animal models 11,12 . However, controversy still exists regarding whether adult hippocampal neurogenesis persists in aged humans, although remarkable efforts have been attempted, including using different antibodies, shortening the intervals of postmortem sampling, and improving the fixation conditions and procedures for immunostaining 2,5,7,8,13-15. Recently, another round of debate was ignited between two labs ${ }^{3,6}$. Despite the conflicting results, they both reached a consensus that new technologies, such as single-cell RNA-seq, will provide new insights in this field, and reveal novel markers for immature neurons and adult NSCs.

\section{AHN exists in aged humans and macaques}

Thus, we first surveyed several samples from adult and aged humans (aged 52, 67, 78, and 85 years, Supplementary Table1) using the classical immature neuronal marker DCX and an optimized staining protocol. More DCX-positive cells (Y67 and Y85, Fig. 1a and Extended Data Fig. 1b) or fewer cells (Y52 and Y78, Extended Data Fig. 1a, c) were detected in each sample, implying remarkable individual variability in AHN. We also observed coexpression of the NSC markers with proliferation markers (Fig. 1b, c), indicating the existence of active NSCs in aged humans. We next traced the proliferation of NSCs and adult neurogenesis by performing consecutive EdU and BrdU labeling in adult and aging Macaca mullata (Y4, Y10, and Y23) (Extended Data Fig. 1d, Supplementary Table1). EdU, BrdU and PAX6 were used to evaluate the continuous proliferation of adult NSCs. We detected NSCs expressing Pax6 that incorporated both EdU and BrdU, even in 23-year-old macaques (Fig. 1d-f, Extended Data Fig. 1g), indicating that these adult NSCs entered S phase twice in the experimental time window. Regarding newborn neurons, we observed many DCX/PSA-NCAM double-positive cells, although the number decreased substantially in aged macaques (Extended Data Fig. 1e). Some $\mathrm{DCX}^{+} \mathrm{EdU}^{+}$and $\mathrm{NeuN}^{+} \mathrm{EdU}^{+}$cells were also identified, which strongly indicated that these cells were newly generated by mitosis (Fig. 1g, h, Extended Data Fig. 1f, h). Overall, NSCs in aged macaques proliferate continuously and produce immature granular cells, although the neurogenesis rate is decreased compared with samples obtained at younger stages. 
a
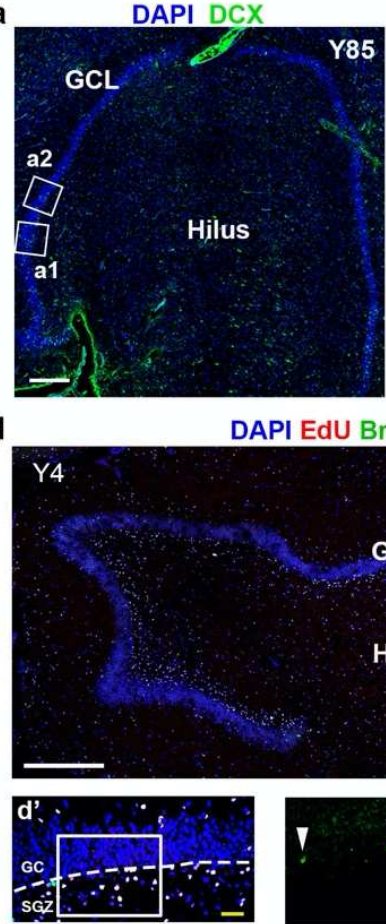

DAPI EdU BrdU PAX6
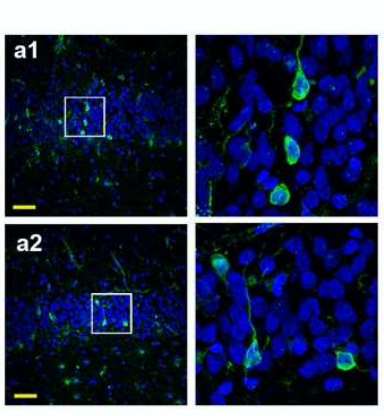

GCL.

Hilus
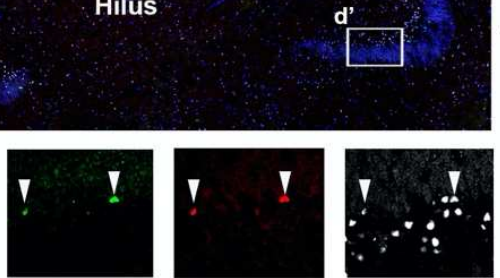

h
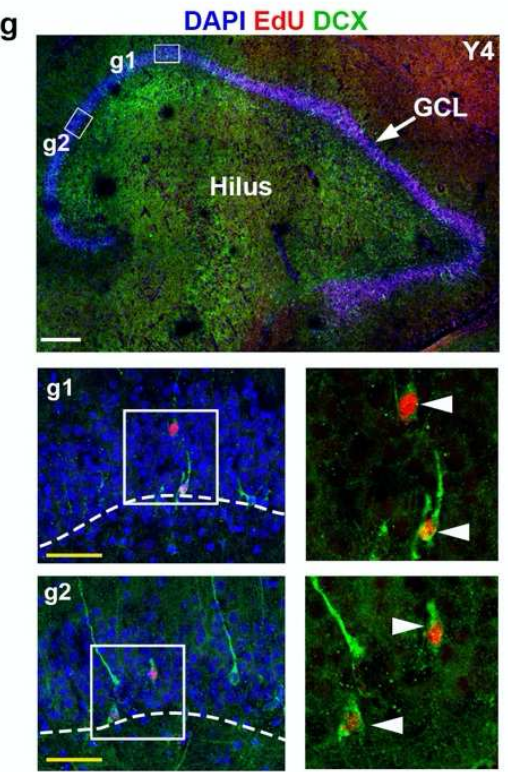

h DAPI EdU DCX
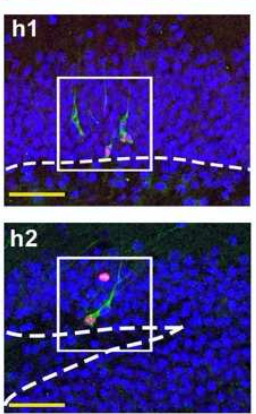

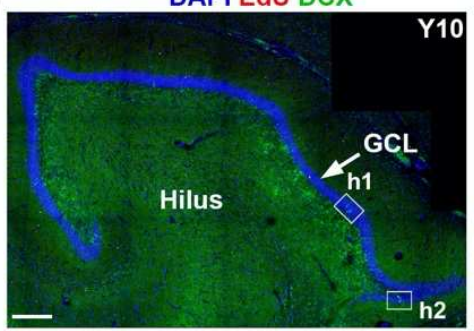

b

DAPI SOX2 MKI67
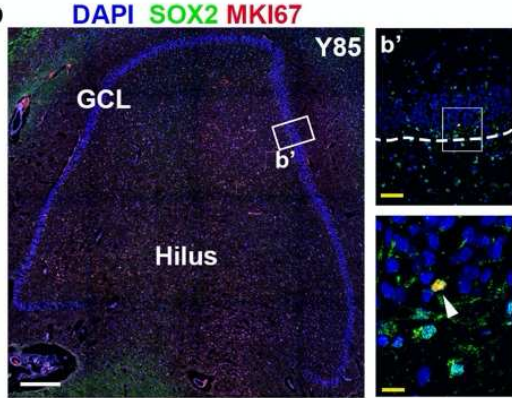

DAPI EdU BrdU PAX

e

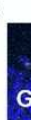

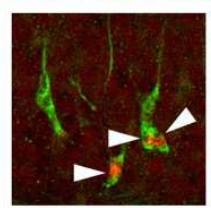

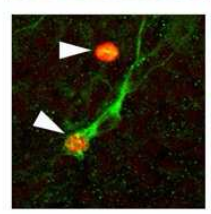

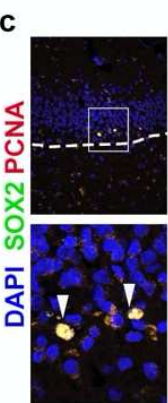

U PAX6
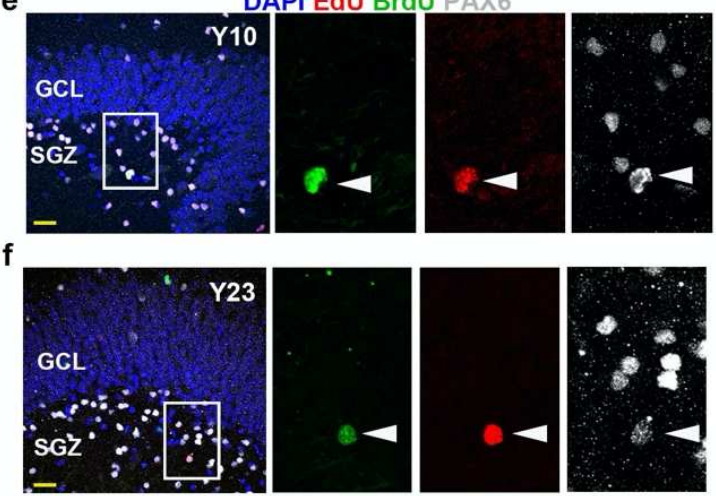

DAPI EdU DCX
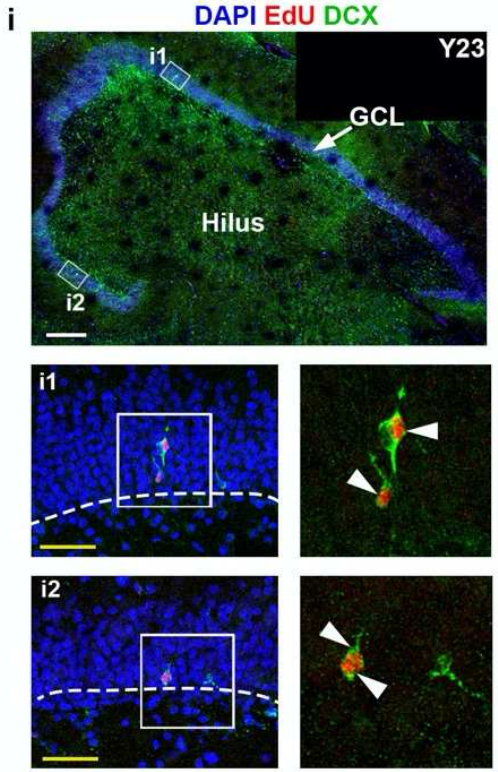

Fig. 1| Adult hippocampal neurogenesis persists in the DG of aged humans and macaques.

a, Expression of the immature neuronal marker DCX was detected in the DG of the hippocampus of aged brains. b, c, Expression of the NSC marker SOX2 and the cell cycle markers MKI67 (b) and PCNA (c) in the DG of the hippocampus of humans aged 85 years. The arrowheads indicate SOX2/MKI67 positive cells in B and SOX2/PCNA positive cells in C. d-e, Adult NSCs in the DG of the hippocampus undergo consecutive proliferation in macaques, as evidenced by EdU/BrdU/PAX6 triple staining. The arrowheads indicate EdU/BrdU/Pax6 triple-positive cells. g-i, New neurons are persistently generated in the DG of the hippocampus in adult and aged macaques, as illustrated by EdU labeling and DCX staining. The arrowhead in each insert shows the colocalization of EdU and DCX. Scale bar in white: $500 \mu \mathrm{m}$, Scale bar in yellow: $50 \mu \mathrm{m}$. 
Next, we aimed to elucidate how hippocampal neurogenesis is regulated from postnatal to aging stages and pinpoint the underlying mechanism of the decreased proliferation capacity of adult NSCs. We conducted sampling and sequencing of 13 macaque hippocampi from postnatal to aging stages (Supplementary Table1). Among the 13 samples, 8 samples containing both cornu ammonis (CA) and dentate gyrus (DG) regions were sequenced; the CA and DG subregions were sequenced separately in the remaining samples (Extended Data Fig. $2 \mathrm{a}, \mathrm{b})$. In total, 132,524 nuclei with a median of 2203 genes detected per nucleus were collected after stringent quality control and removal of the doublets, and all nuclei from the 13 samples were integrated after batch effect removal to preserve biological variation (Extended Data Fig. $2 \mathrm{a}, \mathrm{b})$. Unsupervised clustering analysis and uniform manifold approximation and projection (UMAP) visualization were performed, and 13 major cell types were identified based on the transcriptomic differentially expressed genes (DEGs) and cell-type unique markers reported previously ${ }^{16-18}$ (Fig. 2a, b, Extended Data Fig. 2c, and Supplementary Table2).

The DG and the CA subregions contain distinct cell types and have different physiological functions ${ }^{16,19}$. In addition to the unique cell types, for instance, pyramidal neurons (CA1-3) in the CA regions, granule cells $(\mathrm{GC})$ and immature neurons $(\mathrm{ImmN})$ and adult NSCs in the DG subregions, other major cell types (Astrocyte, OPC, Olig, GABA, and Microglia) exist in both subregions (Fig. 2a). Since adult NSCs (residing in the DG region) and astrocytes (residing in both the CA and DG regions) share very highly similar transcriptome profiles, these cells were clustered in the same group (NSC/Astrocyte) (Fig. 2a, b). We first separated the NSC/Astrocyte clusters from the CA and DG regions. The cells from the two regions showed a region-specific expression profile. DEGs indicated that MEIS2 was a marker for CA astrocytes (Extended Data Fig. 3a-d, Supplementary Table3). This result was confirmed with immunostaining, and we found that most GFAP-positive astrocytes in the CA regions were MEIS2-positive but not cells in the DG region (Extended Data Fig. 3e).

We next analyzed the NSC/Astrocyte residing in the DG. Three subclusters with distinct gene expression profiles were identified (Fig. 2c, e, Supplementary Table4). According to the DEGs, the clusters were annotated as Astrocyte, NSC1, and NSC2. Mature astrocytic markers, such as GFAP, AQP4, CD44 and ITGB4, were preferentially expressed in the Astrocyte cluster. The NSC1 subpopulation was delineated by the dominant expression of the well-documented quiescent NSC markers MFGE8 and PDGFRB, while the NSC2 subcluster prominently expressed ASCL1, PROX1, NES, and VIM, which represented active NSCs (Fig. 2e). The distribution of the three subpopulations in each sample showed that the proportion of 

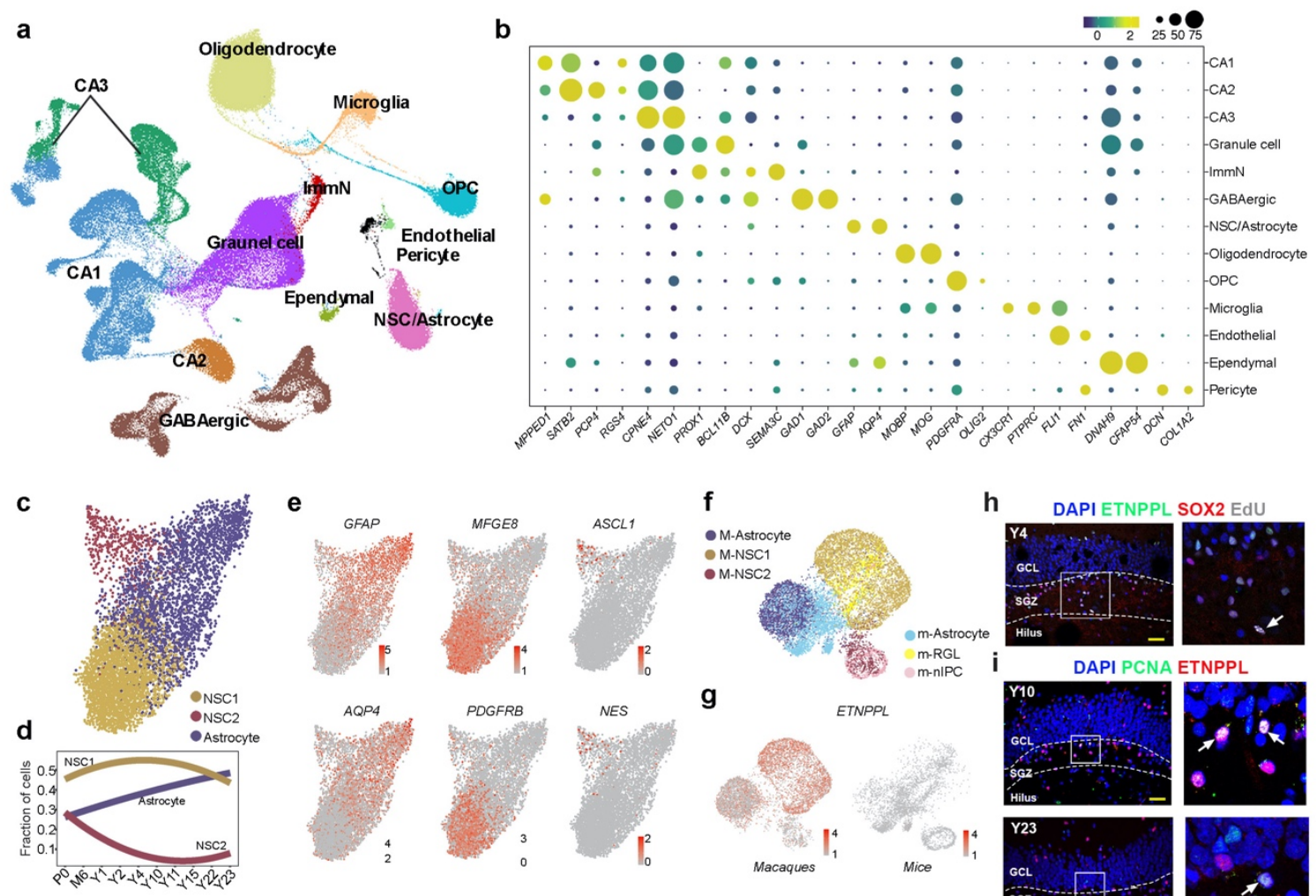

h DAPI ETNPPL SOX2 EdU
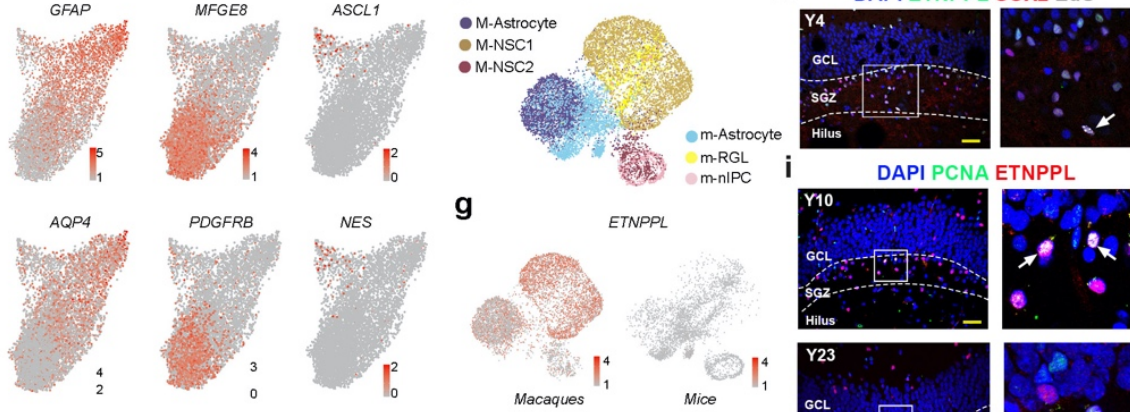

g
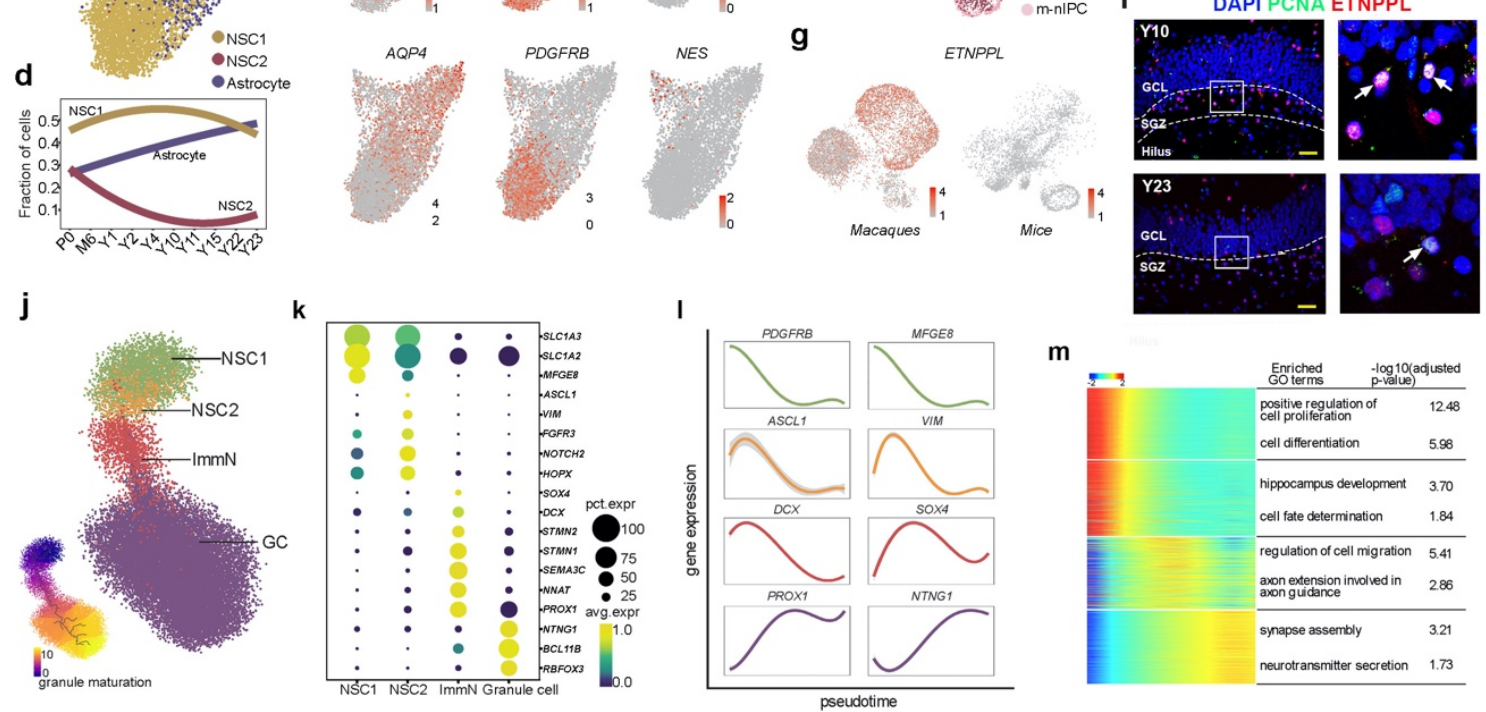

m
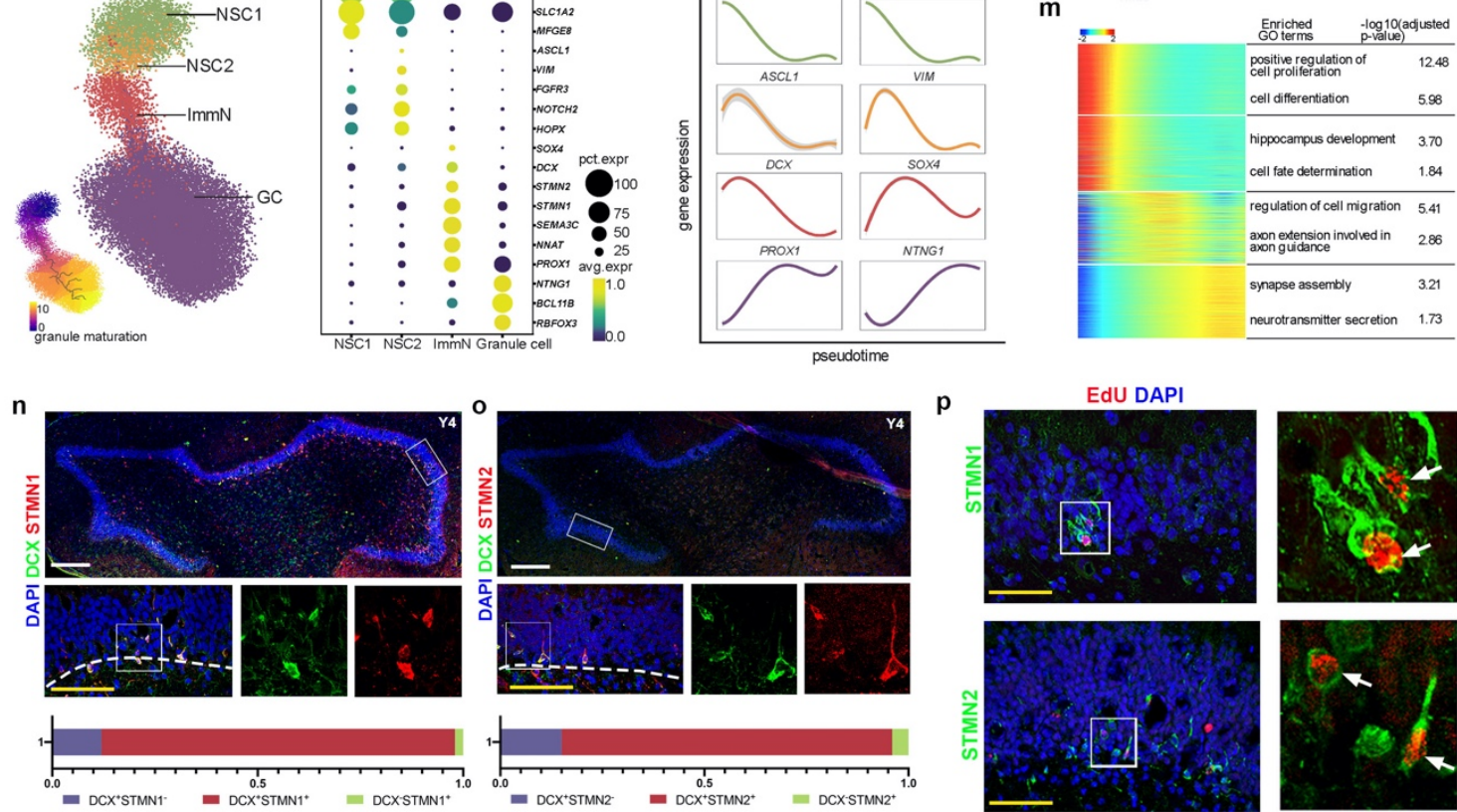

Fig. 2| Transcriptional profiles of the macaque hippocampi at single-nucleus resolution.

a, Clustering of individual hippocampal cells from macaques of different ages via UMAP. Cells are colored by cluster annotation. $\mathbf{b}$, Dot plot showing the average expression of marker genes for the 13 main cell types in Panel a. CA, cornu ammonis; ImmN, immature neuron; NSC/Astrocyte, neural stem cell/astrocyte; OPC, oligodendrocyte progenitor. c, Visualization of NSC/astrocyte subclusters using UMAP. d, Fitted curves show the fraction of cells from three NSC/astrocyte subclusters at different time points. e, The expression of differentially expressed genes among the three distinct NSC/astrocyte subclusters was projected onto UMAP. Cells are colored according to the gene expression levels (red, high; gray, low). f, Integration analysis of astrocytes and adult neural stem cells in macaques with corresponding cell types in mice. g, Featureplot 
showing ETNPPL expression in macaques (right panel) but not in mice (left panel). h, Immunostaining showing ETNPPL colocalization with SOX2 and EdU in 4-year-old brain tissue. The arrow indicates ETNPPL/SOX2/EdU triple positive cell. i, ETNPPL+PCNA+ cells in the SGZ of the hippocampi of 10year-old (top panel) and 23-year-old (bottom panel) macaques. The arrows indicate ETNPPL/PCNA double positive cells. $\mathbf{j}$, The developmental trajectory of the neurogenic lineage is visualized using UMAP. The pseudotime analysis of individual cells is also shown (bottom left panel). $\mathbf{k}$, Dot plot showing the average expression of differentially expressed genes among different cell types in Fig. $2 \mathrm{j}$. l, Fitted curve showing the expression of representative genes over pseudotime. m, Heatmap illustrating the expression of genes that covary across pseudotime. The enriched GO terms are also shown. n,o, Immunostaining analysis showing STMN1/DCX (86\%) and STMN2/DCX (81\%) double-positive cells. p, The colocalization of STMN1 and STMN2 with EdU indicates that these cells are generated via mitosis. The arrows indicate the STMN1/EdU or STMN2/EdU positive cells. Scale bar in white: $500 \mu \mathrm{m}$, scale bar in yellow: $50 \mu \mathrm{m}$.

active NSC2 decreased, indicating decreased proliferation in the adult and aged brains (Fig. 2d). Surprisingly, we observed that the ratio of NSC1 cells, namely, quiescent stem cells, was stable across the lifespan.

Next, we would like to address whether primate NSCs exhibited shared and distinct features to the well-studied rodent models. We compared our dataset with the dataset containing information on postnatal stages in mice ${ }^{17}$. Cells in the Astrocyte and NSC1 clusters matched perfectly with mouse astrocytes and RGL, respectively. Additionally, NSC2 partially merged with nIPC in mice, which strengthened that NSC2 was more active (Fig. 2f, Extended Data Fig. 4a-c). Then, the expressional features of macaque NSCs and mouse RGLs were compared. Most expressed genes were conserved between macaques and mice (4584/5416), 203 genes were highly enriched in mice, and 629 genes were prominently abundant in macaques (Extended Data Fig. 4d, Supplementary Table5). By assessing the expression of the 629 genes in the Allen Brain RNA-ISH Database, we found that 25 of these genes were undetectable in the DG region of the mouse hippocampus (Supplementary Table5), which provided a reservoir of macaque-specific NSC signatures (Extended Data Fig. 4d). For instance, the expression of ETNPPL (ethanol phosphate phospholyase, an enzyme involved in lipid metabolism) was markedly detected in NSCs of macaques but was not expressed in mice at either the RNA or protein levels (Fig. 2f, g, Extended Data Fig. 4d-f). The expression of ETNPPL was readily observed in the SGZ of adult and aged macaques. We validated that ETNPPL was an NSC marker in macaques by performing double or triple immunostaining with SOX2, EdU, and the proliferation marker PCNA (Fig. 2h, i, Extended Data Fig. 4g, h).

With the identified NSCs, we next investigated the developmental process and regulatory continuum of postnatal and adult neurogenesis in macaques. The neurogenic lineage-related clusters (NSC1, NSC2, ImmN, and GC) were integrated for further analysis. Representative markers for each cell type were highlighted (Fig. 2j, k). The trajectory inference with monocle3 showed that NSC1 transited to NSC2, followed by ImmN, and then differentiated into mature 
GC (Fig. 2j). The expression of DEGs along the pseudotime plot exactly recapitulated the regulatory continuum of the process of adult neurogenesis (Fig. 21). An analysis of enriched GO terms validated the biological process and function of each cell type, such as 'cell proliferation' for NSC, 'cell migration' and 'axon extension' for ImmN, and 'neurotransmitter secretion' and 'synapse assembly' for mature GC (Fig. 2m).

\section{STMN1 and STMN2 are new markers for immature granule cells}

Among the genes enriched in the ImmN cluster, STMN1 and STMN2, two members of the Stathmin family, were specifically and predominantly expressed together with the classical markers $D C X$ and $P R O X 1$ (Fig. 2k, 1). The expression of STMN1 or STMN2 was validated with immunostaining. In the newborn samples, abundant STMN1- and STMN2-positive cells were detected, which colocalized with DCX and PSA-NCAM (Extended Data Fig. 5a, b). We precisely identified the characteristics of these two markers by further verifying their expression in samples from 4-year-old animals. First, the colocalization analysis showed that STMN1 or STMN2 exhibited an over $80 \%$ overlap with DCX and PSA-NCAM, which implied the reliability of revealing immature granule cells (Fig. 2n, o, Extended Data Fig. 5b). Some $\mathrm{STMN}^{+}$or $\mathrm{STMN}^{+}$cells were colabeled with Calb2, a transit-expressing newborn-neuron marker ${ }^{20}$ (Extended Data Fig. 5c, d). Some STMN1 ${ }^{+}$or STMN2 ${ }^{+}$cells were also EdU-positive (Fig. 2n, o) but were not positive for either SOX2 or NEUROD1 (Extended Data Fig. 5e-h), suggesting that STMN1 and STMN2 merely labeled postmitotic neurons generated from adult NSCs through mitosis. Furthermore, colabeling of STMN1 or STMN2 with NeuN, a late expressed neuronal marker, showed that $73 \%$ of STMN1 $1^{+}$cells and $62 \%$ of STMN $2^{+}$cells were NeuN-negative (Extended Data Fig. 5i, j), suggesting that these two genes were selectively expressed by nascent neurons. The time-course analysis revealed a substantial decrease in the number of STMN1- and STMN2-positive immature granule cells in aged samples (Extended Data Fig. 5k, 1). In summary, we determined the specific developmental continuum of adult hippocampal neurogenesis at the single-cell level and ascertained that adult neurogenesis continues in aged macaques. We also detected a reservoir of macaque-specific signatures in NSCs and validated STMN1 and STMN2 as reliable immature granule cell markers.

\section{Neuroinflammation contributes to the declined AHN in aged macaques}

A broad body of evidence indicates that adult neurogenesis and the quiescence of adult NSCs are sophistically orchestrated by both intrinsic and extrinsic factors ${ }^{21,22}$. Niche cells provide the major source of extrinsic regulators. Among them, astrocytes play fundamental roles in the proliferation and maintenance of adult NSCs ${ }^{23-26}$. We subgrouped astrocytes into 3 distinct 

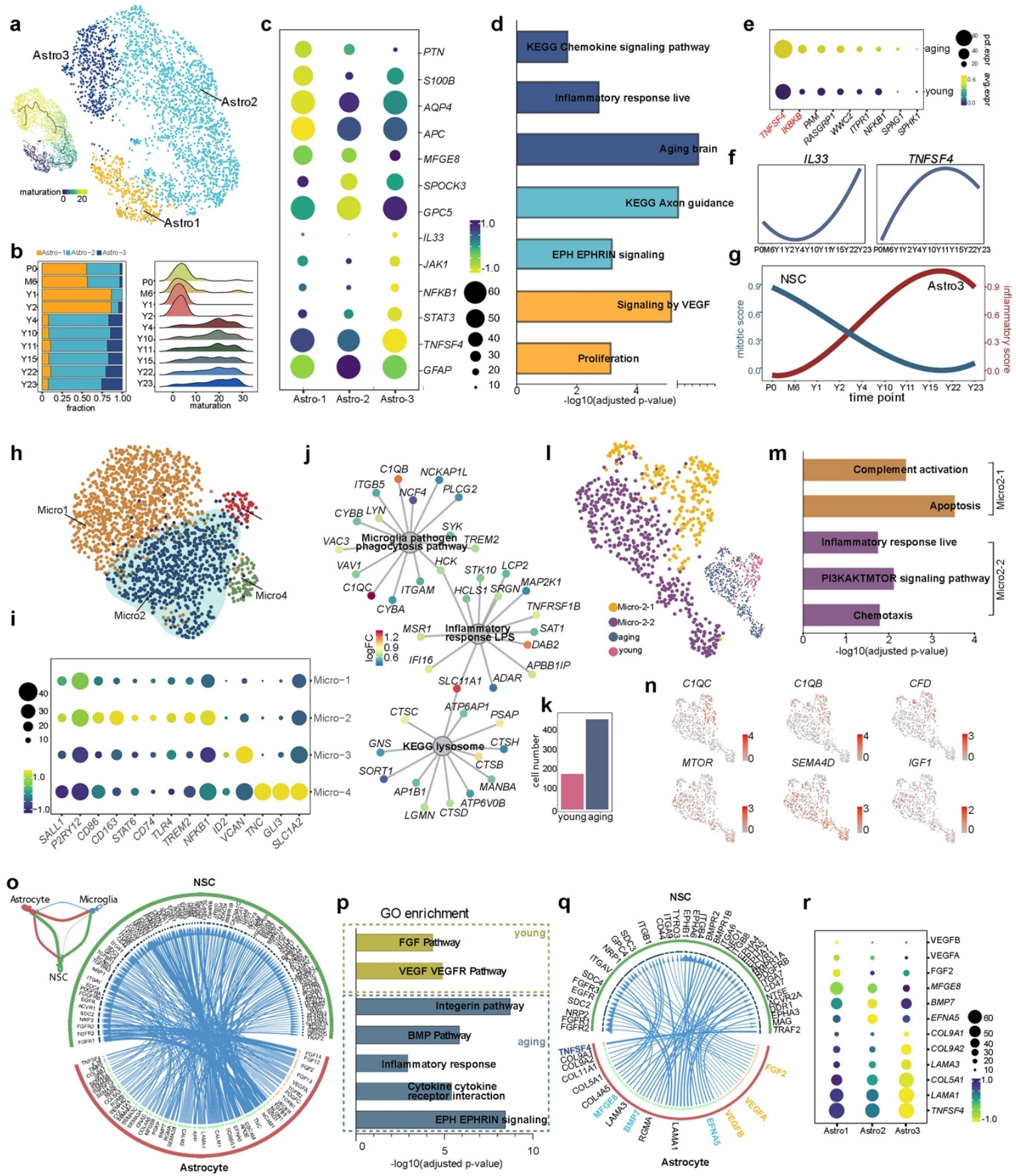

Fig. 3| Transcriptional heterogeneity of cells derived from macaque astrocytes and microglia

a, UMAP showing the clustering of astrocytes with cells colored by astrocyte subclusters (right panel) and pseudotime (left panel). b, Fraction of cells in three distinct astrocyte subclusters at different time points is shown in the bar plot (left panel); the distribution of pseudotime values at each time point is visualized in a ridge plot (right panel). c, Dot plot displaying the average expression of differentially expressed genes among three astrocyte subclusters. d, Gene Ontology enrichment analysis shows the cell properties of three astrocyte subclusters. e, Dot plot showing the expression of chemokine signaling pathway- and inflammatory response-related genes in young (P0-Y2) and aging (Y4-Y23) samples. f, Smoothed relative expression of the inflammatory-related genes IL33 and TNFSF4 over pseudotime. g, Graph showing the smoothed mitotic score and inflammatory score at different time points for NSCs and Astro3 cells. h, UMAP showing the clustering of microglia cells. i, Dot plot showing the average expression of differentially expressed genes among 4 different microglial clusters. $\mathbf{j}$, Network plot of enriched Gene Ontology terms in Micro2. Nodes 
for genes were colored by $\log 2$ fold change. $\mathbf{k}$, Bar plot showing the total number of Micro2 cells from young (P0-Y2) and aging (Y4-Y23) samples. l, Subclusters of activated Micro2 microglia. Cells are colored by cell type (left panel) and age (right panel). m, Enriched Gene Ontology terms in Micro2-1 and Micro2-2. n, UMAP showing the expression of genes related to complement-dependent synaptic pruning and the inflammatory response. o, Network plot showing the ligand-receptor interaction strength between each of the two different cell types (upper left panel). Circos plot illustrating the ligand-receptor interactions between astrocytes and NSCs. p, Bar plot showing the Gene Ontology enriched terms of ligands and receptors identified in Fig. 3o. q, Circos plot showing the interactions between ligands and receptors that were present in the enriched GO terms. $\mathbf{r}$, Dot plot showing the average expression of identified ligands across three astrocyte subclusters.

clusters to investigate the transcriptome dynamics and putative function of astrocytes in the DG of the hippocampus (Fig. 3a). Young samples were predominantly enriched in Astro1 and Astro2, while adult and aging samples were abundant in Astro2 and Astro3 (Fig. 3b). The maturation analysis indicated that astrocytes from young stages (P0-Y2) were less mature than those from older stages (Y4-Y23) (Fig. 3b). DEGs and GO term enrichment analyses indicated that Astro1 was prominently associated with VEGF signaling and proliferation function, and Astro2 entailed mature astrocyte functions, such as axonal guidance. Astro3 was an active astrocyte population that is responsible for chemokine signal transduction and the inflammatory response, expressing $N F K B 1, J A K 1$, STAT3, IL33, etc. (Fig. 3c, d, Extended Data Fig. 6). Older samples manifested a more inflammatory signature than younger samples, and inflammatory-related gene expression increased in the older samples (Fig. 3e, f). Then, we calculated the mitotic score for the NSCs and the inflammatory score for the astrocytes. We observed a concurrence of increased inflammation in astrocytes and decreased proliferation of adult NSCs between Y2 and Y4 (Fig. 3g), at which point neurogenesis decreased dramatically (Extended Data Fig. 5k, 1). This result strongly indicated that environmental inflammation may exert an important function in slowing the mitotic activity of NSCs, as reported in the V-SVZ of mice 27 .

Another type of key niche cell that also plays roles in inflammatory responses in the brain is microglia. We first subset microglial cells residing in the DG subregion but not in the CA subregion (Extended Data Fig. 7a-c). Then, the microglia in the DG were subclustered into 4 populations with distinct gene expression profiles (Fig. 3h, Extended Data Fig. 7d). Micro1 represented a resting state of microglia expressing $P 2 R Y 12, S A L L 1$, etc. Micro2 was a group of active microglia expressing CD74, CD86, CD163, TLR4, and NFKB1 at high levels. Micro3 and Micro4 were two small population (Fig. 3i). The analysis of enriched GO terms showed that Micro2 was associated with the phagocytosis pathway, inflammatory response and lysosome pathways (Fig. 3j). Moreover, over two-thirds of the Micro2 cells were from the aging samples (Fig. 3k). Active microglia exert beneficial functions via phagocytosis or detrimental functions by secreting cytotoxic cytokines ${ }^{28-32}$. To detail the nature of Micro2, it 
was divided into two subclusters, Micro2-1 and Micro2-2 (Fig. 31). The Micro2-1 subcluster mainly containing young samples exhibited anti-inflammatory signatures, such as complement activation and apoptosis, which might be associated with synaptic pruning and phagocytosis of debris from apoptotic cells during early postnatal stages. Micro2-2 subcluster populated with aging samples were involved in the inflammatory response and PI3K/AKT/mTOR pathway activation, which is reportedly related to aging-associated microglial activation (Fig. 31-n). Therefore, our data suggested that active microglia exert different functions at different stages: at the early stage, they positively regulate the development of the hippocampus via the phagocytic pathway, whereas at the elderly stage, active microglia mainly play a proinflammatory role.

Since the microglia and astrocytes were activated in aged samples, we next wanted to address how niche cells regulate adult NSCs. We outlined the potential interaction between niche cells and adult NSCs by investigating the ligand-receptor interaction with the iTALK package ${ }^{33}$. We compared the interaction strength among microglia, astrocytes and NSCs, and the results showed that astrocytes and NSCs had a stronger interaction (Fig. 3o). Thus, we further delineated the potential interactions between astrocytes and NSCs (Fig. 30). The analysis of representative GO terms showed that VEGF-VEGFR signaling and the FGF pathway were enriched in the young samples (P0-Y2), and the Integrin pathway, BMP pathway, Eph-Ephrin signaling, and cytokine-receptor interaction were enriched in the old samples (Y4Y23) (Fig. 3p, q). Our results were consistent with previous findings that VEGF and FGF are involved in the mechanism by which astrocytes promote adult NSC proliferation ${ }^{21,34}$; the Integrin pathway, BMP signaling and Eph-Ephrin signaling have consistently been shown to be involved in regulating NSC quiescence by niche cells ${ }^{21,22}$. Therefore, at a young stage, astrocytes may increase NSC proliferation, and at an old stage, astrocytes may promote NSC quiescence and inflammation.

We next investigated the expression levels of the ligands in the subclusters of astrocytes. $V E G F A, V E G F B$, and FGF2 were expressed at high levels in Astro1. Genes involved in the quiescence of adult NSCs (e.g., $M F G E 8, B M P 7$, and $E F N A 5$ ) were abundantly enriched in Astro2. In Astro3, the cytokine TNFSF4 might lead to an inflammatory effect on NSCs. Interestingly, we also found that extracellular matrix-related genes, which play an important role in modulating the local inflammatory milieu, as well as homeostasis of NSCs, were enriched in Astro3 (Fig. 3r). Overall, we provided evidence that distinct astrocyte subtypes exerted different effects on modulating adult NSCs, including increasing proliferation (Astro1), maintaining quiescence (Astro2), and inducing an inflammatory response (Astro3). The 

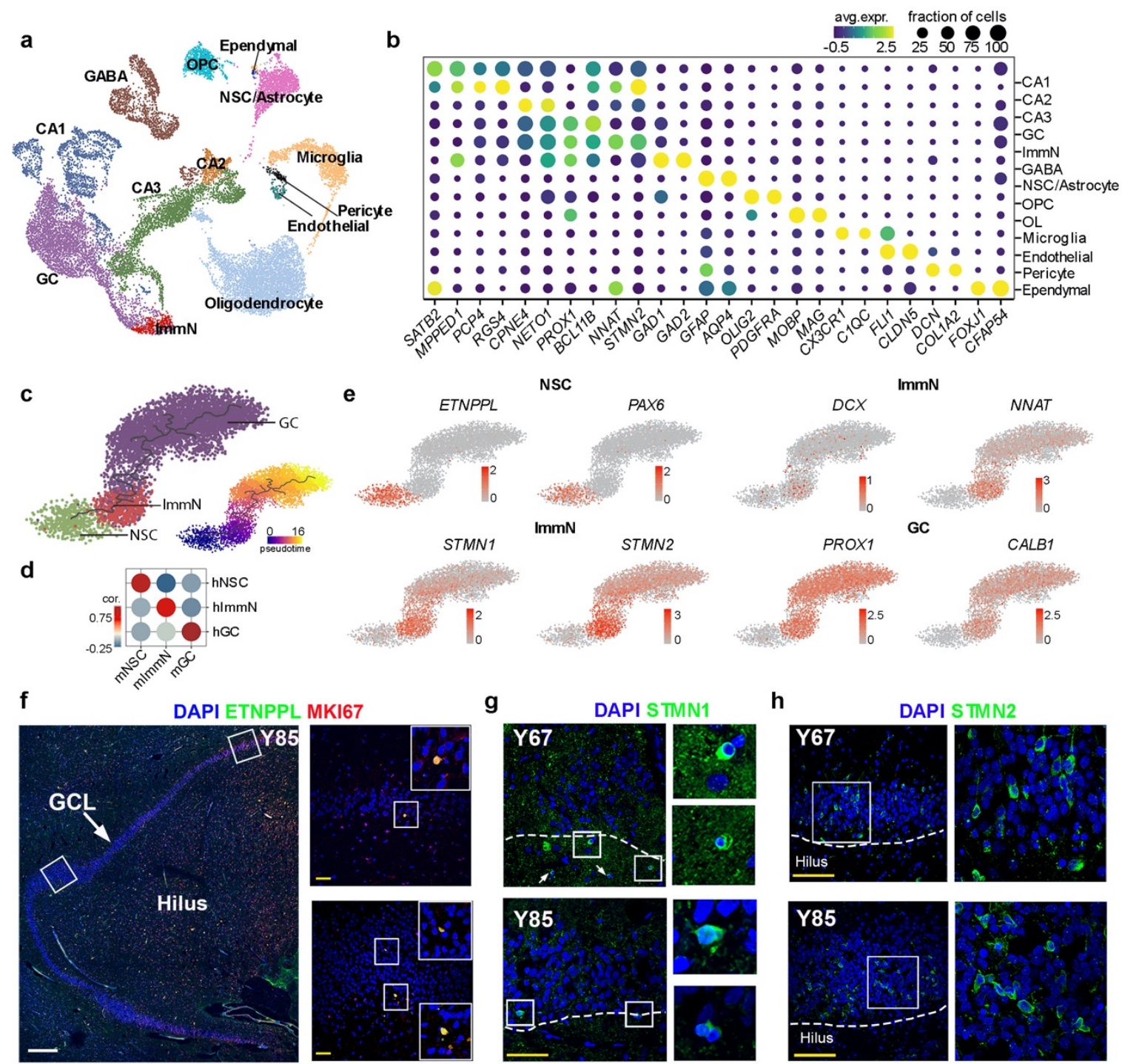

i

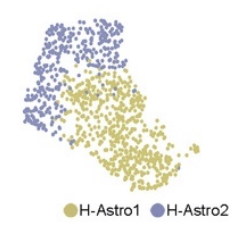

k

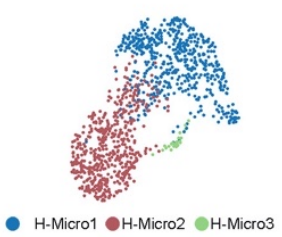

j
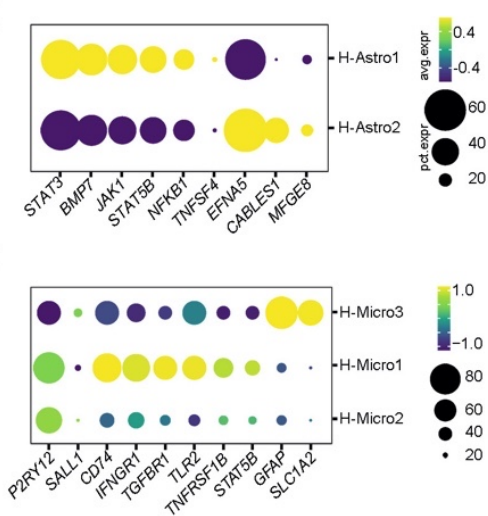

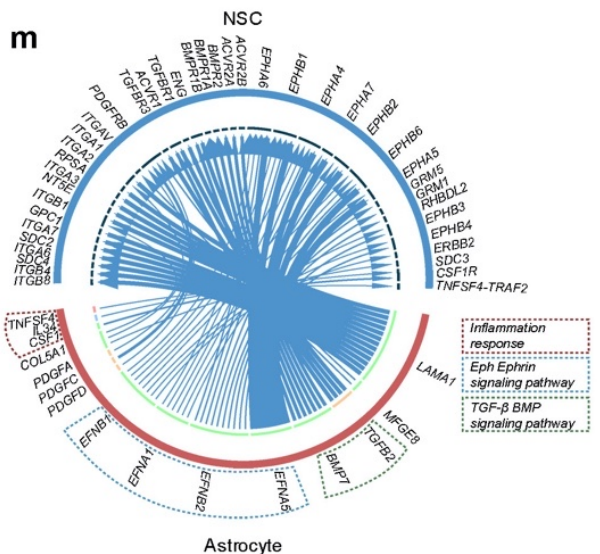

Fig. 4| Persistent adult hippocampal neurogenesis was identified in aged humans. plot showing the mean expression of marker genes for the 13 major cell types. c, Developmental trajectory inference of the neurogenic lineage is visualized with UMAP (left panel); the pseudotime analysis of individual cells is also presented (right panel). d, Correlation analysis of NSC, ImmN, and GC clusters between humans and macaques. e, UMAP showing the expression of representative markers for each cluster shown in Fig. 4c. f, ETNPPL+MKI67+ cells illustrating NSCs in the cell cycle. g, $\mathbf{h}$, Expression of STMN1 (g) and STMN2 (h) in the DG of the hippocampus of humans aged 67 years and 85 years. Scale bar in white: 
$500 \mu \mathrm{m}$, Scale bar in yellow: $50 \mu \mathrm{m}$. i, UMAP showing the clustering of astrocytes in the human dentate gyrus. $\mathbf{j}$, Visualization of the expression of differentially expressed genes across astrocyte subclusters in a dot plot. k, UMAP showing the clustering of microglia in the human dentate gyrus. $\mathbf{l}$, Dot plot showing the expression of differentially expressed genes across microglial subclusters. $\mathbf{m}$, Circos plot illustrating the ligand-receptor interactions between human astrocytes and NSCs.

decreased function of Astro1 and increased functions of Astro2 and Astro3 may contribute to the decline in adult neurogenesis during aging.

\section{Transcription dynamics of hippocampal neurogenesis in aged humans}

To further investigate the adult neurogenesis and the regulatory continuum in aged human hippocampi, four human samples from donors (Y67, Y85, Y87, and Y92) were collected and analyzed using snRNA-seq (Supplementary Table1). In total, 22119 nuclei were obtained and categorized into 13 cell populations (Fig. 4a, Extended Data Fig. 8a-c, and Supplementary Table6). A cohort of ImmN was also revealed in our dataset. Adult NSCs were subset from the NSC/Astrocyte cluster (Extended Data Fig. 9a-c, Supplementary Table7). Lineage trajectory inference showed that ImmN in humans was positioned between NSC and GC, which expressed the immature neuron markers as those in macaque at high levels, including $D C X$, STMN1, STMN2, PROX1, NNAT, SOX4, etc. (Fig. 4c, e, Extended Data Fig. 9d, e). The correlation analysis showed that the human ImmM (H-ImmN) cluster was strongly correlated with the macaque ImmN cluster, indicating evolutionarily conserved transcriptome profiles in primates (Fig. 4c, d). We also observed the specific expression of ETNPPL in human NSCs (Fig. 4e). MKI $67^{+} \mathrm{ETNPPL}^{+}$double-positive cells were detected in the DG of the hippocampus (Fig. 4f), which strongly indicated that ETNPPL was a primate-specific NSC marker. The expression of STMN1 and STMN2 was also verified in aged human samples (Fig. 4g, h). The colocalization of STMN1 and STMN2 with CALB2 but not with NEUROD1 strongly indicated that both markers label immature neurons, which confirmed that adult neurogenesis exists in aged human hippocampi (Extended Data Fig. 9f-i).

Last, we examined the NSC niche in aged humans. The astrocytes in humans were subgrouped into two populations, H-Astro1 and H-Astro2 (Fig. 4i). H-Astro1 was enriched in genes related to the inflammatory response, similar to those in macaques (e.g., JAK/STAT pathway, $N F K B 1$, and TNFSF4), which strengthened the hypothesis that a group of astrocytes was activated during brain aging (Fig. 4j). H-Astro2 expressed genes related to regulating NSC quiescence (e.g., $P D G F A / B / C, M F G E 8$, and Ephrin family). We integrated the two datasets to investigate whether the regulation of active astrocytes was conserved between humans and macaques. River-plot showed that active astrocytes (M-Astro3 and H-Astro1) were conserved between macaques and humans (Extended Data Fig.10a, b). Likewise, the analysis of microglia 
in aged humans also revealed a cluster of active microglia that correlated strongly with the active microglia in macaques and expressed CD74, IGNGR1, TLR2, and TNFRSF1B (Fig. 4k, 1, Extended Data Fig. 10c, d). The interaction of astrocytes and adult NSCs revealed conserved pathways between humans and macaques, such as the EPH-EPHRIN pathway, BMP pathway, and inflammatory signaling pathway. However, in the Eph-Ephrin pathway, EFNA5 was the only ligand enriched in macaques, while $E F N B 1, E F N B 2$, and $E F N A 1$ were also significantly expressed in humans, indicating conserved pathways but distinct preferred ligand-receptor counterparts between humans and macaques (Fig. $4 \mathrm{~m}$ ).

\section{Discussion}

In this study, we systematically survey the molecular and cellular dynamics of the hippocampi in macaques across the lifespan and in aged humans using high-throughput snRNA-seq. In the neurogenic lineage, immature granule cells are readily revealed with unsupervised cluster method in both macaques and aged humans. The developmental and expressional continuum from adult NSCs to immature and mature GCs is delineated. We reveal a trail of NSC signatures and validate ETNPPL is a primate-specific marker for NSCs. We also verify that STMN1 and STMN2 as new markers to detect immature granule cells in the hippocampus of primates. Strong evidence indicates that the hippocampal neurogenesis persists in aged humans and macaques, albeit the number of immature neurons declined.

In the niche cells, we illustrate a cluster of active astrocytes and a cluster of microglia exhibiting proinflammatory signature. The interaction strength among microglia, astrocytes, and NSCs show that astrocytes and NSCs have a stronger interaction, implying that active astrocytes play essential roles on NSCs. The transcriptome dynamics of astrocytes and the analysis of the interaction of astrocytes with NSCs infer that distinct astrocyte subtypes exert distinct functions by providing distinct signals to adult NSCs. Our findings highlight the importancy of astrocytes during the adult hippocampal development and neurogenesis. The correlation of decreased neurogenesis with increased neuroinflammation implies that manipulating the neuroinflammatory status of the brain might be a feasible approach to restore adult hippocampal neurogenesis in aged individuals and individuals with disease.

$1 \quad$ Paredes, M. F. et al. Does Adult Neurogenesis Persist in the Human Hippocampus? Cell Stem Cell 23, 780-781, doi:10.1016/j.stem.2018.11.006 (2018).

2 Sorrells, S. F. et al. Human hippocampal neurogenesis drops sharply in children to undetectable levels in adults. Nature 555, 377-381, doi:10.1038/nature25975 (2018).

3 Sorrells, S. F. et al. Positive Controls in Adults and Children Support That Very Few, If Any, New Neurons Are Born in the Adult Human Hippocampus. $J$ Neurosci 41, 2554-2565, doi:10.1523/JNEUROSCI.0676-20.2020 (2021).

4 Kempermann, G. et al. Human Adult Neurogenesis: Evidence and Remaining Questions. Cell Stem Cell 23, 25-30, doi:10.1016/j.stem.2018.04.004 (2018). 


\begin{tabular}{|c|c|c|}
\hline $\begin{array}{l}376 \\
377 \\
378\end{array}$ & 5 & $\begin{array}{l}\text { Moreno-Jimenez, E. P. et al. Adult hippocampal neurogenesis is abundant in neurologically healthy } \\
\text { subjects and drops sharply in patients with Alzheimer's disease. Nat Med 25, 554-560, } \\
\text { doi:10.1038/s41591-019-0375-9 (2019). }\end{array}$ \\
\hline 379 & 6 & Moreno-Jimenez, E. P., Terreros-Roncal, J., Flor-Garcia, M., Rabano, A. \& Llorens-Martin, M. \\
\hline 380 & & ividences for Adult Hippocampal Neurogenesis in Humans. $J$ Neurosci 41, 2541-2553, \\
\hline 381 & & oi:10.1523/JNEUROSCI.0675-20.2020 (2021). \\
\hline 382 & 7 & Boldrini, M. et al. Human Hippocampal Neurogenesis Persists throughout Aging. Cell Stem Cell 2 \\
\hline 383 & & 589-599 e585, doi:10.1016/j.stem.2018.03.015 (2018). \\
\hline 384 & 8 & Tobin, M. K. et al. Human Hippocampal Neurogenesis Persists in Aged Adults and Alzheimer's Disease \\
\hline 385 & & Patients. Cell Stem Cell 24, 974-982 e973, doi:10.1016/j.stem.2019.05.003 (2019). \\
\hline 386 & 9 & Navarro Negredo, P., Yeo, R. W. \& Brunet, A. Aging and Rejuvenation of Neural Stem Cells and Their \\
\hline 387 & & Niches. Cell Stem Cell 27, 202-223, doi:10.1016/j.stem.2020.07.002 (2020). \\
\hline 388 & 10 & Bartsch, T. \& Wulff, P. The hippocampus in aging and disease: From plasticity to vulnerability. \\
\hline 389 & & Neuroscience 309, 1-16, doi:10.1016/j.neuroscience.2015.07.084 (2015). \\
\hline 390 & 11 & Toda, T. \& Gage, F. H. Review: adult neurogenesis contributes to hippocampal plasticity. Cell Tissue Res \\
\hline 391 & & 373, 693-709, doi:10.1007/s00441-017-2735-4 (2018). \\
\hline 392 & 12 & Abbott, L. C. \& Nigussie, F. Adult neurogenesis in the mammalian dentate gyrus. Anat Histol Embryol \\
\hline 393 & & 49, 3-16, doi:10.1111/ahe.12496 (2020). \\
\hline 394 & 13 & Cipriani, S. et al. Hippocampal Radial Glial Subtypes and Their Neurogenic Potential in Human Fetuse \\
\hline 395 & & nd Healthy and Alzheimer's Disease Adults. Cereb Cortex 28, 2458-2478, doi:10.1093/cercor/bhy09 \\
\hline 396 & & 2018). \\
\hline 397 & 14 & Dennis, C., Suh, L., Rodriguez, M., Kril, J. \& Sutherland, G. Human adult neurogenesis across the age \\
\hline 398 & & in immunohistochemical study. Neuropathology and applied neurobiology 42, 621-638 (2016). \\
\hline 399 & 15 & Flor-Garcia, M. et al. Unraveling human adult hippocampal neurogenesis. Nat Protoc 15, 668-693, \\
\hline 400 & & oi:10.1038/s41596-019-0267-y (2020). \\
\hline 401 & 16 & Cembrowski, M. S., Wang, L., Sugino, K., Shields, B. C. \& Spruston, N. Hipposeq: a comprehensive \\
\hline 402 & & RNA-seq database of gene expression in hippocampal principal neurons. Elife 5, e14997, \\
\hline 403 & & doi:10.7554/eLife.14997 (2016). \\
\hline 404 & 17 & Hochgerner, H., Zeisel, A., Lonnerberg, P. \& Linnarsson, S. Conserved properties of dentate gyrus \\
\hline 405 & & neurogenesis across postnatal development revealed by single-cell RNA sequencing. Nat Neurosci 21, \\
\hline 406 & & 90-299, doi:10.1038/s41593-017-0056-2 (2018). \\
\hline 407 & 18 & Miller, J. A. et al. Conserved molecular signatures of neurogenesis in the hippocampal subgranular zone \\
\hline 408 & & f rodents and primates. Development 140, 4633-4644, doi:10.1242/dev.097212 (2013). \\
\hline 409 & 19 & Bond, A. M., Ming, G. L. \& Song, H. Adult Mammalian Neural Stem Cells and Neurogenesis: Five \\
\hline 410 & & Decades Later. Cell Stem Cell 17, 385-395, doi:10.1016/j.stem.2015.09.003 (2015). \\
\hline 411 & 20 & Brandt, M. D. et al. Transient calretinin expression defines early postmitotic step of neuronal \\
\hline 412 & & differentiation in adult hippocampal neurogenesis of mice. Mol Cell Neurosci 24, 603-613, \\
\hline 413 & & doi:10.1016/s1044-7431(03)00207-0 (2003). \\
\hline 414 & 21 & Vicidomini, C., Guo, N. \& Sahay, A. Communication, Cross Talk, and Signal Integration in the Adult \\
\hline 415 & & Hippocampal Neurogenic Niche. Neuron 105, 220-235, doi:10.1016/j.neuron.2019.11.029 (2020). \\
\hline 416 & 22 & Urban, N., Blomfield, I. M. \& Guillemot, F. Quiescence of Adult Mammalian Neural Stem Cells: A \\
\hline 417 & & Highly Regulated Rest. Neuron 104, 834-848, doi:10.1016/j.neuron.2019.09.026 (2019). \\
\hline 418 & 23 & Garber, C. et al. Astrocytes decrease adult neurogenesis during virus-induced memory dysfunction via \\
\hline 419 & & IL-1. Nat Immunol 19, 151-161, doi:10.1038/s41590-017-0021-y (2018). \\
\hline 420 & 24 & Casse, F., Richetin, K. \& Toni, N. Astrocytes' Contribution to Adult Neurogenesis in Physiology and \\
\hline 421 & & Alzheimer's Disease. Front Cell Neurosci 12, 432, doi:10.3389/fncel.2018.00432 (2018). \\
\hline 422 & 25 & Ashton, R. S. et al. Astrocytes regulate adult hippocampal neurogenesis through ephrin-B signaling. Nat \\
\hline 423 & & Neurosci 15, 1399-1406, doi:10.1038/nn.3212 (2012). \\
\hline 424 & 26 & Song, H., Stevens, C. F. \& Gage, F. H. Astroglia induce neurogenesis from adult neural stem cells. Nature \\
\hline 425 & & $417,39-44(2002)$ \\
\hline 426 & 27 & Kalamakis, G. et al. Quiescence Modulates Stem Cell Maintenance and Regenerative Capacity in the \\
\hline 427 & & Aging Brain. Cell 176, 1407-1419 e1414, doi:10.1016/j.cell.2019.01.040 (2019). \\
\hline 428 & 28 & Masuda, T., Sankowski, R., Staszewski, O. \& Prinz, M. Microglia Heterogeneity in the Single-Cell Era. \\
\hline 429 & & Cell Rep 30, 1271-1281, doi:10.1016/j.celrep.2020.01.010 (2020). \\
\hline 430 & 29 & Paolicelli, R. C. et al. Synaptic pruning by microglia is necessary for normal brain development. science \\
\hline 431 & & 333, $1456-1458(2011)$ \\
\hline 432 & 30 & Sato, K. Effects of microglia on neurogenesis. Glia 63, 1394-1405 (2015). \\
\hline 433 & 31 & Ekdahl, C. T. Microglial activation-tuning and pruning adult neurogenesis. Frontiers in pharmacology \\
\hline 434 & & 3, $41(2012)$ \\
\hline 435 & 32 & Sierra, A. et al. Microglia shape adult hippocampal neurogenesis through \\
\hline
\end{tabular}


phagocytosis. Cell stem cell 7, 483-495 (2010).

33 Wang, Y. et al. iTALK: an R package to characterize and illustrate intercellular communication. BioRxiv, 507871 (2019).

34 Shetty, A. K., Hattiangady, B. \& Shetty, G. A. Stem/progenitor cell proliferation factors FGF-2, IGF-1, and VEGF exhibit early decline during the course of aging in the hippocampus: role of astrocytes. Glia 51, 173-186 (2005). 


\section{Macaque samples collection}

446 Macaque monkeys were raised and received cares in the non-human primate center of the Institute of 447 Biophysics, Chinese Academy of Sciences (IBP, CAS, Supplementary Table1). All procedures were 448 conducted in accordance with the Principles for the Ethical Treatment of Non-Human Primates and were approved by the Institutional Animal Care and Use Committee of the Institute of Biophysics, CAS.

\section{Human samples collection}

451

452

453

454

455

456

Postmortem human brain samples (Supplementary Table1) from individuals without neurological disorders were provided by the National Human Brain Bank for Development and Function, Chinese Academy of Medical Sciences and Peking Union Medical College, Beijing, China. All sample collection and experimental procedures were followed national laws and international ethical and technical guidelines.

We collected tissue blocks from samples with a shorter post-mortem interval. Blocks were either frozen in liquid nitrogen or fixed overnight in $4 \%$ PFA at $4^{\circ} \mathrm{C}$. Frozen blocks were used for single nucleus RNA-seq. Fixed blocks were cryoprotected using a 30\% sucrose solution and then embedded in OCT. Embedded tissue was cut on a Leica CM1950 very low temperature freezer into $20 \mu \mathrm{m}$ coronal sections, and mounted on glass slides for immunohistochemistry analysis.

\section{Consecutive EdU and BrdU labeling}

To trace the proliferation of NSCs and adult neurogenesis, EdU (Thermo Fisher Scientific, A10044) and BrdU (Thermo Fisher Scientific, B23151) labeling was conducted. Briefly, EdU labeling ( $5 \mathrm{mg} / \mathrm{kg}$ body weight) was administered to macaques intravenously (i.p.) twice a week for 10 weeks. With a two-monthinterval, BrdU was intravenously administered $(10 \mathrm{mg} / \mathrm{kg})$ twice a week for 10 weeks. Then, the animals were sacrificed 2 months of post-administration.

For macaque brain sample preparation, adult and senile macaque monkeys were anesthetized with Katamine (10 mg/kg body weight) and perfused with cold artificial cortico-spinal fluid (ACSF). The whole brains were rapidly dissected on ice. For single nucleus RNA-seq, the hippocampal formations and other brain regions in the right hemisphere were isolated and quick frozen in liquid nitrogen. For the immunostaining, the left part of each brain was cut coronally into around $1 \mathrm{~cm}$-thickn slabs and fixed in 4\% PFA for $12 \mathrm{~h}$, following cryoprotected in a sucrose gradient from $10 \%, 20 \%$ to $30 \%$, and then embed the tissue with OCT. 
475 Frozen sections were equilibrated to room temperature (RT) and rinsed with PBST buffer $(0.3 \%$

476 Triton-X in PBS) for $10 \mathrm{~min}$. To reduce the remaining aldehyde groups, the sections were 477 incubated with a $0.5 \%$ sodium triacetoxyborohydride (NaBH(OAc)3; Merck, 316393) solution

478 for $30 \mathrm{~min}$ at room temperature. Next, the samples were boiled in a sodium citrate buffer (10 $\mathrm{mM}$ sodium citrate tribasic dihydrate, $\mathrm{pH}$ 6.0) for 20 minutes, and cooled down to room temperature. For PSA-NCAM antibody staining, we conducted conduct antigen retrieval in Tris-EDTA buffer (50 mM Tris, $1 \mathrm{mM}$ EDTA, pH9.0). After antigen retrieval, samples were washed with PBST for 10 min and then blocked with a 5\% normal donkey serum solution (NDS) (JACKSON, 017-000-121T) for $1 \mathrm{~h}$ at room temperature. Subsequently, samples were incubated for three to five days at $4{ }^{\circ} \mathrm{C}$ with $5 \%$ normal donkey serum containing the primary antibodies (Supplementary Table8). The secondary antibodies incubation and DAPI nuclear staining were performed at room temperature for at least $2 \mathrm{~h}$. Autofluorescence Eliminator reagent $(0.1 \%$ Sudan Black in $70 \%$ ethanol) was applied to the aged samples to eliminate lipofuscin autofluorescence. After three washes with PBST, sections were mounted with Fluoromount-G (SouthernBiotech, 0100-01) and stored at $4^{\circ} \mathrm{C}$ in the dark.

To visualize EdU-labelled cells, a Click-iT EdU Imaging Kit (Invitrogen, C10639) was used according to manufacturer's guidelines. For BrdU staining, the tissue was incubated with $2 \mathrm{M} \mathrm{HCl}$ at RT for 20 minutes, followed by sequential incubation with $5 \%$ NDS, the anti-BrdU antibody and secondary antibody, respectively.

\section{Imaging}

Serial coronal sections of the hippocampus were collected from macaques and humans. Images were acquired using an Olympus FV3000 confocal microscope with a 30x oil immersion objective (NA 1.30). Tiled images were acquired and stitched using Olympus FluoView software. Image processing and analysis were conducted using FLUOVIEW FV3000 and Fiji/ImageJ software and compiled with Adobe Photoshop 2020.

\section{Single nuclei isolation, $10 \times$ Genomics Chromium, and sequencing}

Nuclei were isolated from fresh-frozen tissue as previously described with some modifications and improvements. Briefly, tissue samples were minced into pieces $<5 \mathrm{~mm}$ and then homogenized using a glass dounce tissue grinder (Sigma, Cat\# D8938) in $2 \mathrm{ml}$ of Nuclei EZ lysis buffer (Sigma, Cat\# NUC-101) on ice. After two incubations on ice (with $4 \mathrm{~mL}$ of lysis buffer and 5 min each time), the homogenate was filtered through a $70 \mu \mathrm{m}$ strainer, and then we used Debris Removal Solution (Miltenyi Biotech, \#130-109398) to perform density gradient centrifugation to clean the nuclear suspension according to the manufacturer's protocol. Isolated nuclei were resuspended and washed with nuclei suspension buffer (NSB, 
consisting of $1 \times \mathrm{PBS}, 0.1 \% \mathrm{BSA}$ and $0.4 \mathrm{U} / \mu \mathrm{l}$ Ambion $^{\mathrm{TM}}$ RNase inhibitor (Thermo Fisher, Cat\# AM2684)) and filtered through a $35 \mu \mathrm{m}$ cell strainer. Nuclei were counted using a hemocytometer and diluted to 1000 nuclei $/ \mu \mathrm{L}$ for optimal 10X loading. Approximately 8000 nuclei were targeted and captured for each reaction. Steps from Chip B loading to cDNA library construction was carried out with Chromium Single Cell 3' Reagent Kits $\mathrm{v} 3$ according to the 10X official instructions.

\section{Processing of macaque snRNA-seq data}

A cell-by-gene count matrix was generated following sequence alignment with Cell Ranger software (https://support.10xgenomics.com/). The raw count matrix from each sample was loaded to the scrublet pipeline individually, with the parameter expected_doublet_ratio set to 0.06 to identify possible doublets ${ }^{35}$. A total of 32049/182036 cells with a doublet score greater than the doublet score threshold was considered as doublets and thus discarded from subsequent analysis. The filtered cell-by-gene count matrix was then loaded into the Seurat pipeline ${ }^{36}$ for downstream analysis. Cells that did not meet the following criteria were omitted: 1) cells with a number of detected genes ranging from 800 to $7500 ; 2$ ) cells with nUMI greater than 1000 ; and 3) cells with a percentage of mitochondrial counts less than $1 \%$. Overall, 132524 cells were retained for downstream analysis. Next, the filtered cell-by-gene matrix was imported to function CreateSeuratObject to create a Seurat object followed by log-normalization of the gene expression matrix with function NormalizeData. Then, we identified variable genes that exhibited high cell-to-cell variations using the function FindVariableFeatures and performed a dimension reduction analysis in the space of those variable features with the functions RunPCA and RunUMAP. We removed batch effects and preserved the biological variation present in our dataset by conducting a canonical correction analysis among individual samples with the functions FindIntegrationAnchors and IntegrateData ${ }^{36}$. Clustering analysis was performed with the functions FindNeighbors and FindClusters.

\section{Distinguishing DG and CA cells based on regional transcriptional identities}

We first computed differentially expressed genes between cells obtained from individual dissection of CA and DG for the each of the following individual cell types to distinguish DG and CA cells from whole hippocampal samples: astrocytes, microglia, OPCs, oligodendrocytes and GABAergic neurons. Then, we performed principal component analysis of each cell type in whole hippocampal samples based on the identified regional differentially expressed genes. Next, we fitted the resulting principal components to the k-means algorithm for clustering analysis. Subsequently, we determined the regional characteristics of the identified clusters by calculating the enrichment score for genes enriched in CA and DG across all clusters using the AUCell package ${ }^{37}$. A cluster with a higher enrichment score for DG-enriched genes was considered as a DG-derived group, and a cluster with a higher enrichment score for CA-enriched genes was identified as a CA-derived group.

\section{Construction of the macaque adult neurogenic trajectory}

We first extracted NSCs, ImmNs and granule from the whole dataset and performed the dimension reduction 
analysis using those cells to infer the adult neurogenic trajectory in macaque hippocampi. Then, for trajectory construction, we converted the Seurat object to a SingleCellExperiment object and performed trajectory graph learning analysis and pseudotime measurement with the functions learn_graph and order_cells from monocle $3^{38-40}$, respectively.

\section{Comparison of transcriptional profiles between macaque and mouse NSCs.}

We first assembled cells from our dataset with those from a published scRNA-seq dataset of the mouse dentate gyrus ${ }^{41}$ based on orthologues identified between the macaque and mouse genomes with the $\mathrm{R}$ package biomaRt ${ }^{42}$. Next, we subdivided NSCs from the merged dataset and computed differentially expressed genes between macaque and mouse NSCs with the Seurat function FindMarkers.

\section{Identification of cell-cell communication}

We studied cell-cell communication using iTALK (https://github.com/Coolgenome/iTALK), a package designed to study cell interactions. Briefly, the rawParse function was applied to retain the top $50 \%$ highly expressed genes as input for the FindLR function to identify ligand-receptor pairs between cell types. We mainly focused on the ligand-receptor pairs where ligands were obtained from astrocytes and receptors were expressed in NSCs. The identified interactions were visualized using the NetView and LRPlot functions.

\section{Processing of human snRNA-seq data}

Reads were aligned to the human reference genome hg19 with Cell Ranger software (https://support.10xgenomics.com/), and a cell-by-gene count matrix was then generated. Then, candidate doublets were identified by individually importing the raw count matrix from each human sample into the scrublet software by setting the parameter expected_doublet_ratio to $0.06^{35}$. A total of 2287/26865 cells with a doublet score exceeding the setting threshold were then omitted from the subsequent analysis. The filtered cell-by-gene count matrix was then loaded into the Seurat pipeline. Then, cells that did not meet the following criteria were discarded: 1) cells with more than 200 detected genes and 2) cells with fewer than 10000 detected genes. After removing low-quality cells, 22119 cells were retained for the subsequent analysis. The filtered count matrix was then log-normalized with the NormalizeData function. Next, variable features were computed with the function FindVariableFeatures, and dimension reduction analysis was performed on these variable features with the functions RunPCA and RunUMAP. Batch effects derived from nonbiological variations were removed by conducting a canonical correction analysis among individual samples with the functions FindIntegrationAnchors and IntegrateData. The clustering analysis was carried out with the functions FindNeighbors and FindClusters.

\section{Construction of the human adult neurogenic trajectory}

Similar to the trajectory inference analysis conducted for the macaque dataset, we first extracted NSCs, ImmNs and granule cells from the whole human dataset and then performed the dimension reduction analysis on those cells. Next, we used the UMAP embedding and clusters generated by Seurat as input for the 
trajectory learning analysis and pseudotime measurement analysis with the functions learn_graph and order_cells from monocle3.

\section{Quantification and statistical analysis}

580

581

588

589

590

591

592

593

594

595

596

597

598

599

600

601

602

603

604

605

606

607

608

609

610

611

612

613

614

615

616

617

618

All data obtained from macaques and humans at each age were collected from at least three independent experiments. The error bars represent the s.d. Statistical analyses were performed unpaired two-tailed Student's t-tests and the multiple comparison test after ANOVA using GraphPad Prism software. Sample size and P values are given in the Figure legends.

\section{Data availability}

The single-nucleus RNA-seq data used in this study has been deposited in the Gene Expression Omnibus (GEO) under the accession number GSE163737.

35 Wolock, S. L., Lopez, R. \& Klein, A. M. Scrublet: Computational Identification of Cell Doublets in Single-Cell Transcriptomic Data. Cell Syst 8, 281-291 e289, doi:10.1016/j.cels.2018.11.005 (2019).

36 Stuart, T. et al. Comprehensive Integration of Single-Cell Data. Cell 177, 1888-1902 e1821, doi:10.1016/j.cell.2019.05.031 (2019).

37 Aibar, S. et al. SCENIC: single-cell regulatory network inference and clustering. Nat Methods 14, 10831086, doi:10.1038/nmeth.4463 (2017).

38 Cao, J. et al. The single-cell transcriptional landscape of mammalian organogenesis. Nature 566, 496502, doi:10.1038/s41586-019-0969-x (2019).

39 Qiu, X. et al. Reversed graph embedding resolves complex single-cell trajectories. Nat Methods 14, 979982, doi:10.1038/nmeth.4402 (2017).

40 Trapnell, C. et al. The dynamics and regulators of cell fate decisions are revealed by pseudotemporal ordering of single cells. Nat Biotechnol 32, 381-386, doi:10.1038/nbt.2859 (2014).

41 Hochgerner, H., Zeisel, A., Lonnerberg, P. \& Linnarsson, S. Conserved properties of dentate gyrus neurogenesis across postnatal development revealed by single-cell RNA sequencing. Nat Neurosci 21, 290-299, doi:10.1038/s41593-017-0056-2 (2018).

42 Durinck, S. et al. BioMart and Bioconductor: a powerful link between biological databases and microarray data analysis. Bioinformatics 21, 3439-3440 (2005).

\section{Acknowledgements}

This work was supported by the National Key R\&D Program of China (2019YFA0110100), the Strategic Priority Research Program of the Chinese Academy of Sciences (XDB32010100), the National Basic Research Program of China (2017YFA0102601 and 2017YFA0103303), the National Natural Science Foundation of China (NSFC) (31771140 and 81891001). BUAA-CCMU Big Data and Precision Medicine Advanced Innovation Center Project (BHME-2019001), Collaborative Research Fund of Chinese Institute for Brain Research, Beijing (2020-NKX-PT-02 and 2020-NKX-PT-03). Human tissues were provided by the National Human Brain Bank for Development and Function, Chinese Academy of Medical Sciences and Peking Union Medical College, Beijing, China. This study was supported by the Institute of Basic Medical Sciences, Chinese Academy of Medical Sciences, Neuroscience Center, and the China Human Brain Banking Consortium. 


\section{Author contributions}

621 W.W., X.W., Q.W., and W.J. conceived the project. W.W., and X.W., designed the experiments and wrote the 622 manuscript. M. Y. and W.W. performed immunostaining and imaging. M.W., B.Z., X.J., J. Z., Y. G., and Y. 623 L. analyzed the RNA-seq data. W.W., C.Y., and B. W. prepared the macaque samples. Q.M. and Q.Z. 624 performed snRNA-seq experiments. W.Q., and C.M. prepared and provided the human samples. All authors 625 edited and proofread the manuscript.

626

\section{Competing interests}

628 The authors declare no competing interests.

629

630 
Wei Wang ${ }^{1,5,9}$, Mengdi Wang ${ }^{1,5,9}$, Meng Yang ${ }^{1,5,9}$, Bo Zeng ${ }^{1,5,9}$, Wenying Qiu ${ }^{2,9}$, Qiang Ma ${ }^{1,5}$, Xiaoxi Jing ${ }^{3}$, 635 Qianqian Zhang, ${ }^{1,5}$ Baisong Wang ${ }^{3}$, Chonghai Yin ${ }^{4}$, Jiyao Zhang ${ }^{3}$, Yuxin $\mathrm{Ge}^{3}$, Yufeng $\mathrm{Lu}^{1,5}$, Weizhi $\mathrm{Ji}^{6}$, Qian

1 State Key Laboratory of Brain and Cognitive Science, CAS Center for Excellence in Brain Science and 640 China.

6412 Institute of Basic Medical Sciences, Neuroscience Center, National Human Brain Bank for Development 642 and Function, Chinese Academy of Medical Sciences; Department of Human Anatomy, Histology and 643 Embryology, School of Basic Medicine, Peking Union Medical College, Beijing 100005, China.

6443 State Key Laboratory of Cognitive Neuroscience and Learning, IDG/McGovern Institute for Brain 645 Research, Beijing Normal University, Beijing, 100875, China.

6464 Bioland Laboratory (Guangzhou Regenerative Medicine and Health Guangdong Laboratory), Guangzhou, 647510005 , China.

6485 University of Chinese Academy of Sciences, Beijing 100049, China.

6496 Yunnan Key Laboratory of Primate Biomedical Research, Institute of Primate Translational Medicine, 650 Kunming University of Science and Technology, Kunming, 650500, China.

6517 Institute for Stem Cell and Regeneration, CAS, Beijing 100101, China.

6528 Advanced Innovation Center for Human Brain Protection, Beijing Institute for Brain Disorders, Capital 653 Medical University, Beijing, 100069, China.

6549 These authors contributed equally.

655

656 657

*Correspondence: qianwu@bnu.edu.cn (Q.W.), machao@ibms.cams.cn (C.M.), xiaoqunwang@ibp.ac.cn (X.W.) 

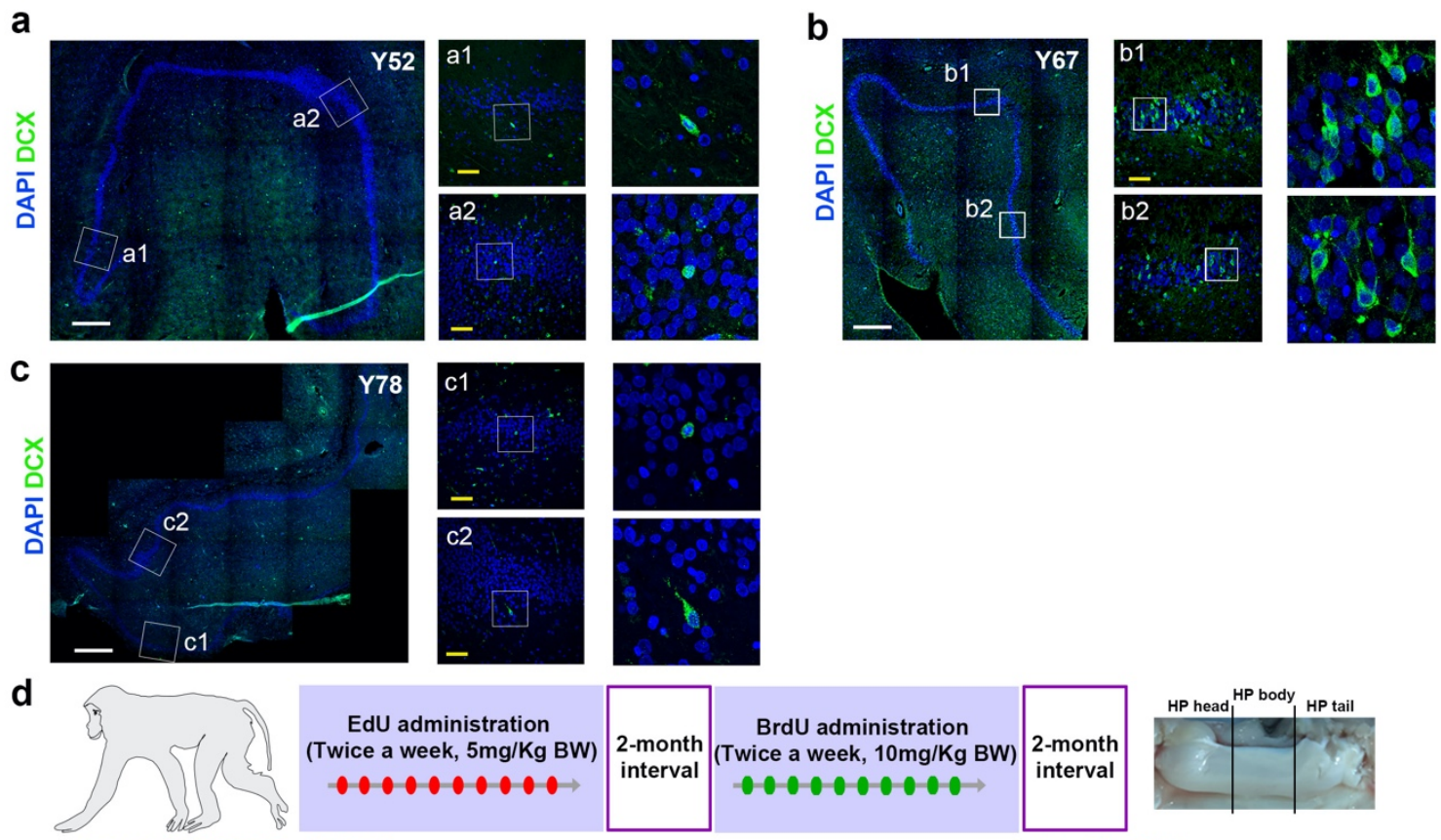

e DAPI DCX PSA-NCAM
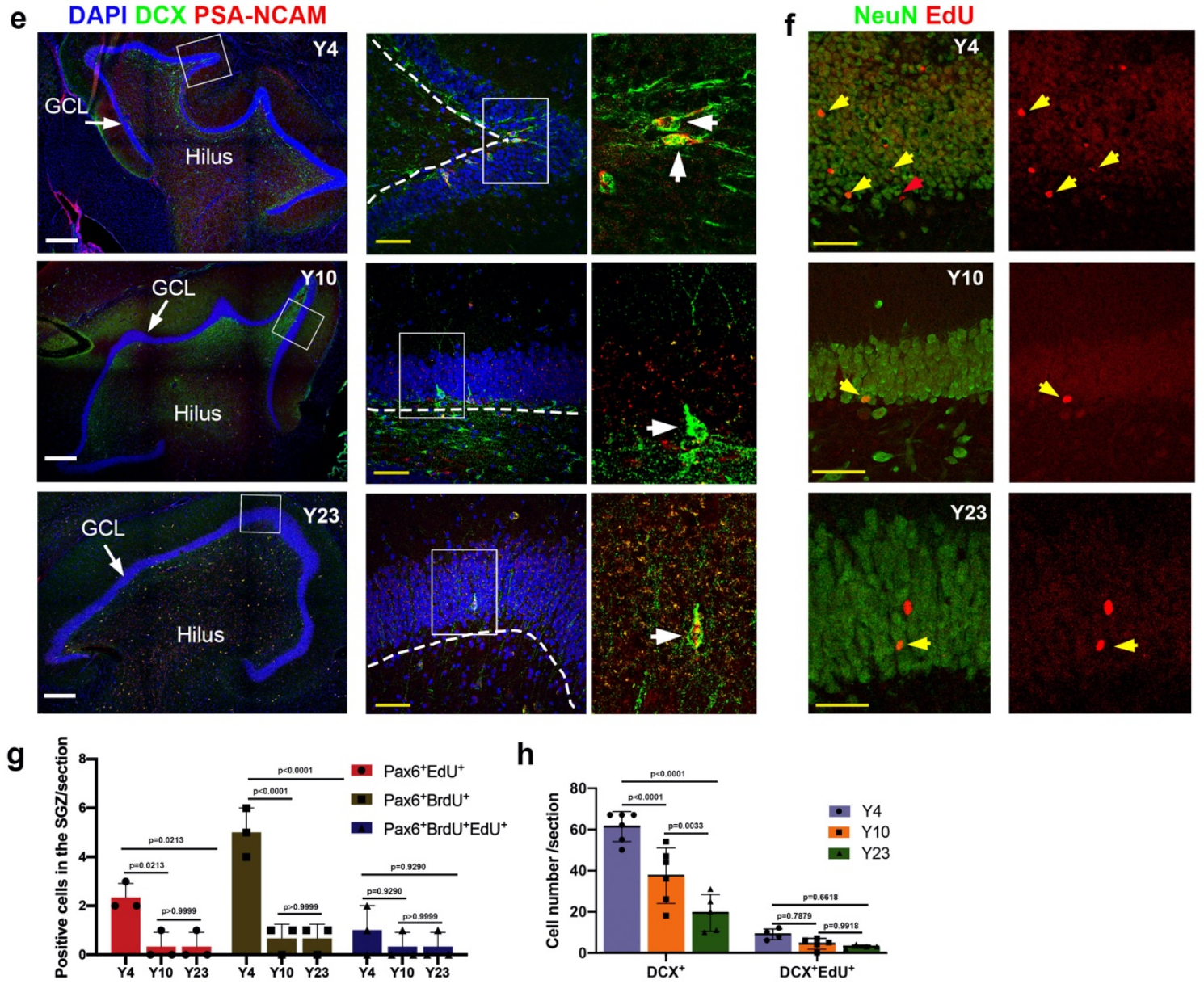

662 Extended Data Fig. 1 |Adult hippocampal neurogenesis persists in the DG of aged humans.

663 a-c, The expression of DCX in the hippocampal DG of 52-year-old (a), 67-year-old (b), and 
78-year-old humans (c). d, Schematic of EdU and BrdU tracing assays in macaques. e, Cryostat 665 sections showing DCX and PSA-NCAM immunostaining in the DG of the hippocampi from 666 macaques aged 4, 10, and 23 years. White arrows in the right column indicate double-positive 667 cells. f, NEUN and EdU cells are visualized in the GCL, and yellow arrows highlight the 668 double-positive cells. g, Quantification of staining for the neural stem cell marker PAX6 with 669 EdU and BrdU. Sidak's multiple comparisons test was used, each dot represents a single 670 experiment. mean \pm s.d. h, Quantification of DCX+ and DCX+EDU+ cells located in the DG 671 of the hippocampus. Sidak's multiple comparisons test was used, each dot represents a single 672 experiment. mean \pm s.d. Scale bar in white: $500 \mu \mathrm{m}$, scale bar in yellow: $50 \mu \mathrm{m}$. 
a
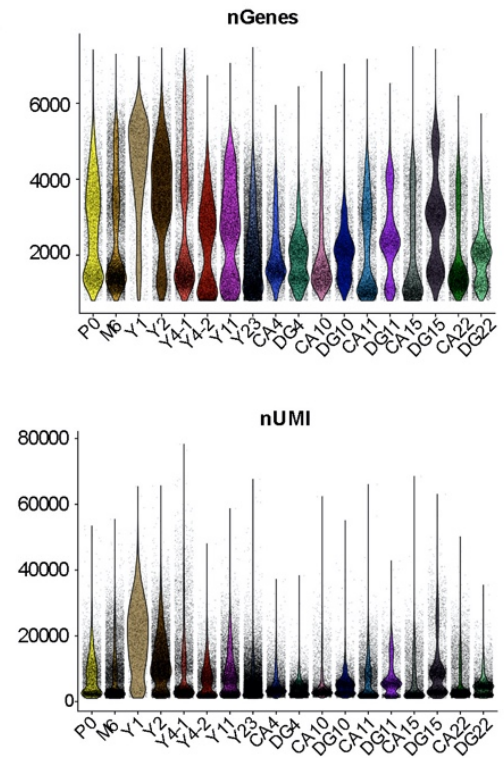

b

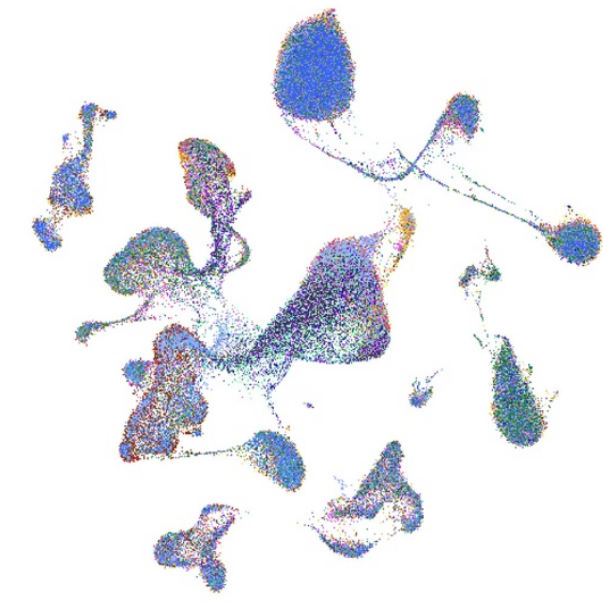

P0 M6 OY1 OY2 OY41 OY42 OY11 OY23 OCA4 ODG4 $\odot$ CA10 - DG10 OCA11 ODG11 OCA15 ODG15 OCA22 DG22

C
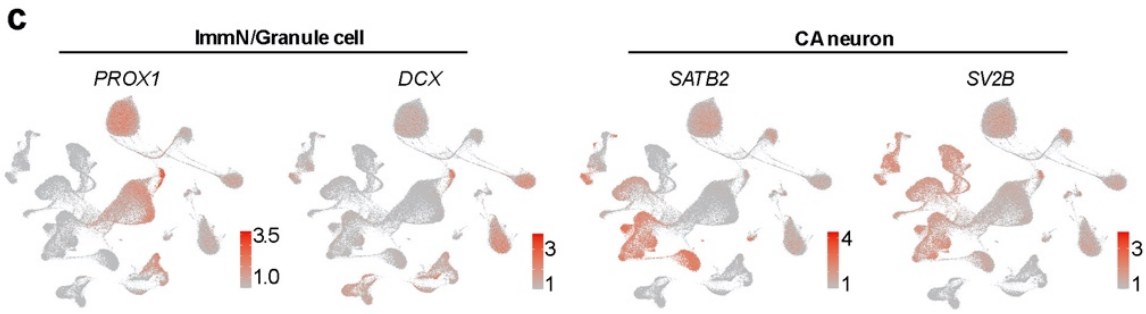

GABAergic

GAD1

\begin{tabular}{llll}
\multicolumn{5}{c}{ NSC/Astrocyte } \\
\hline SLC1A2 & AQP4 & PAX6 & GFAP
\end{tabular}

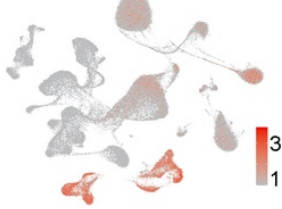

Microglia

CX3CR1
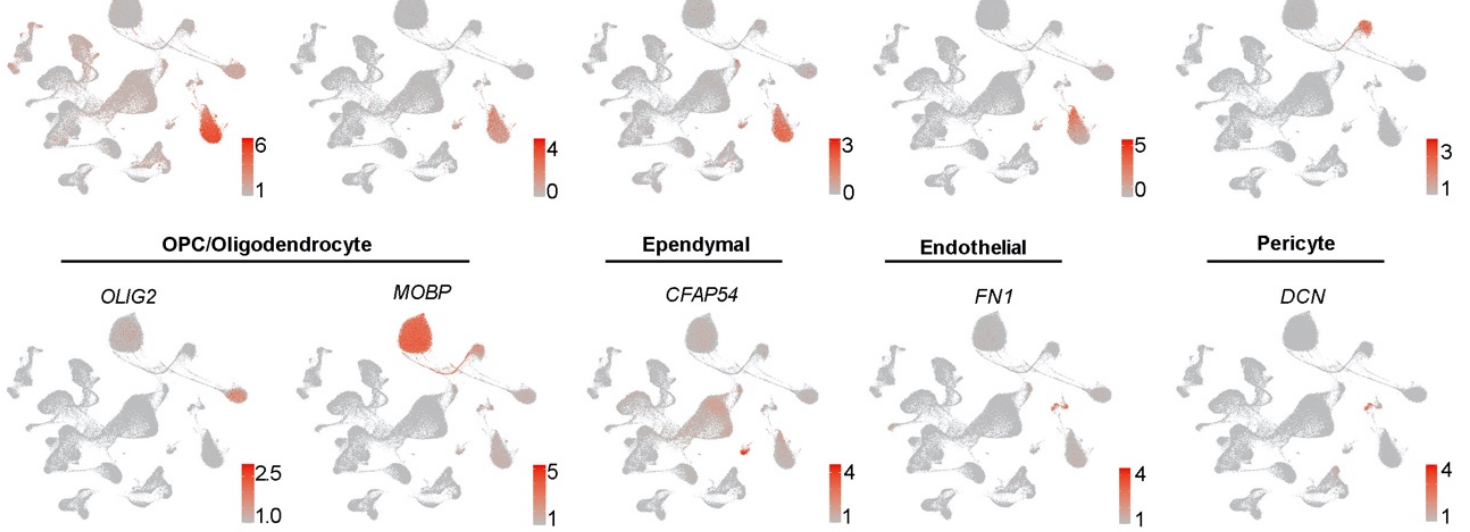

674 Extended Data Fig. 2 | Transcriptional features of macaque hippocampi. a, Quality control metrics for macaque hippocampal samples; each dot represents a single cell. Cells with gene numbers per cell (nGene) $<800$ and $>7500$ (upper panel), as well as nUMI $<1000$ (lower

677 panel), were excluded from the subsequent analysis. b, UMAP showing the cell distribution in

67813 samples. Cells are colored by samples. c, The expression of well-known marker genes for major cell types was visualized using UMAP. 

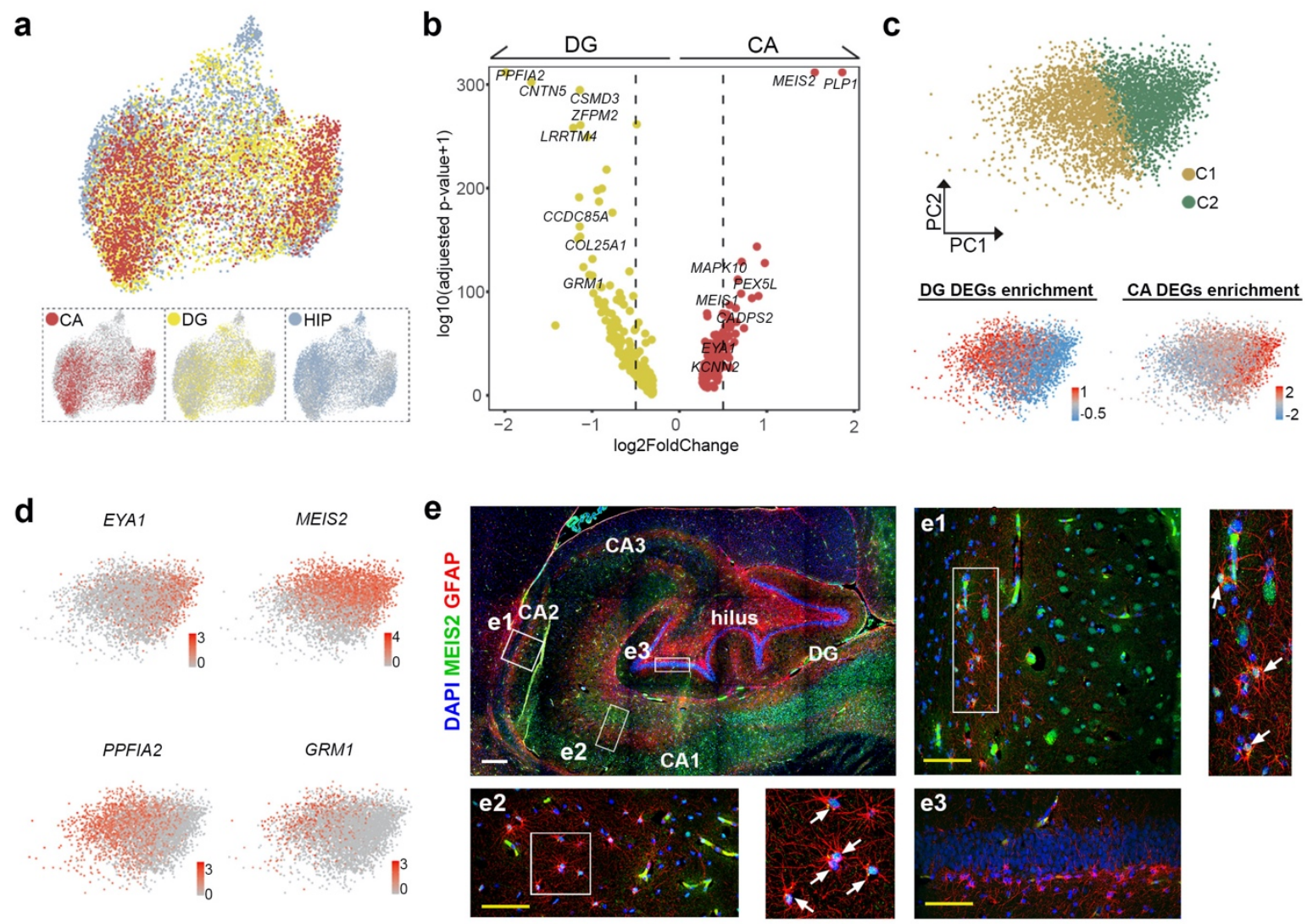

Extended Data Fig. 3 |Regional transcriptional profiles of astrocytes in the macaque hippocampus. a, Visualization of the cell distribution obtained from individual dissection of the CA, DG and whole hippocampus. b, Volcano plot showing the regional differentially expressed genes. c, Clustering of astrocytes from whole hippocampi into two groups via PCA reduction (upper panel). Projection of the enrichment score of the regional differentially expressed genes from CA and DG onto the PCA plot (lower panel). d, PCA plot showing the expression of DG-enriched genes (PPFIA2 and GRIM1) and CA-enriched genes (EYA1 and MEIS2). e, GFAP is expressed in both the CA and DG regions and coexpressed with MEIS2 in the CA region (e1 and e2) but not in the DG region (e3). The arrows indicate the GFAP/MEIS2 double positive cells. Scale bar in white: $500 \mu \mathrm{m}$, scale bar in yellow: $50 \mu \mathrm{m}$. 


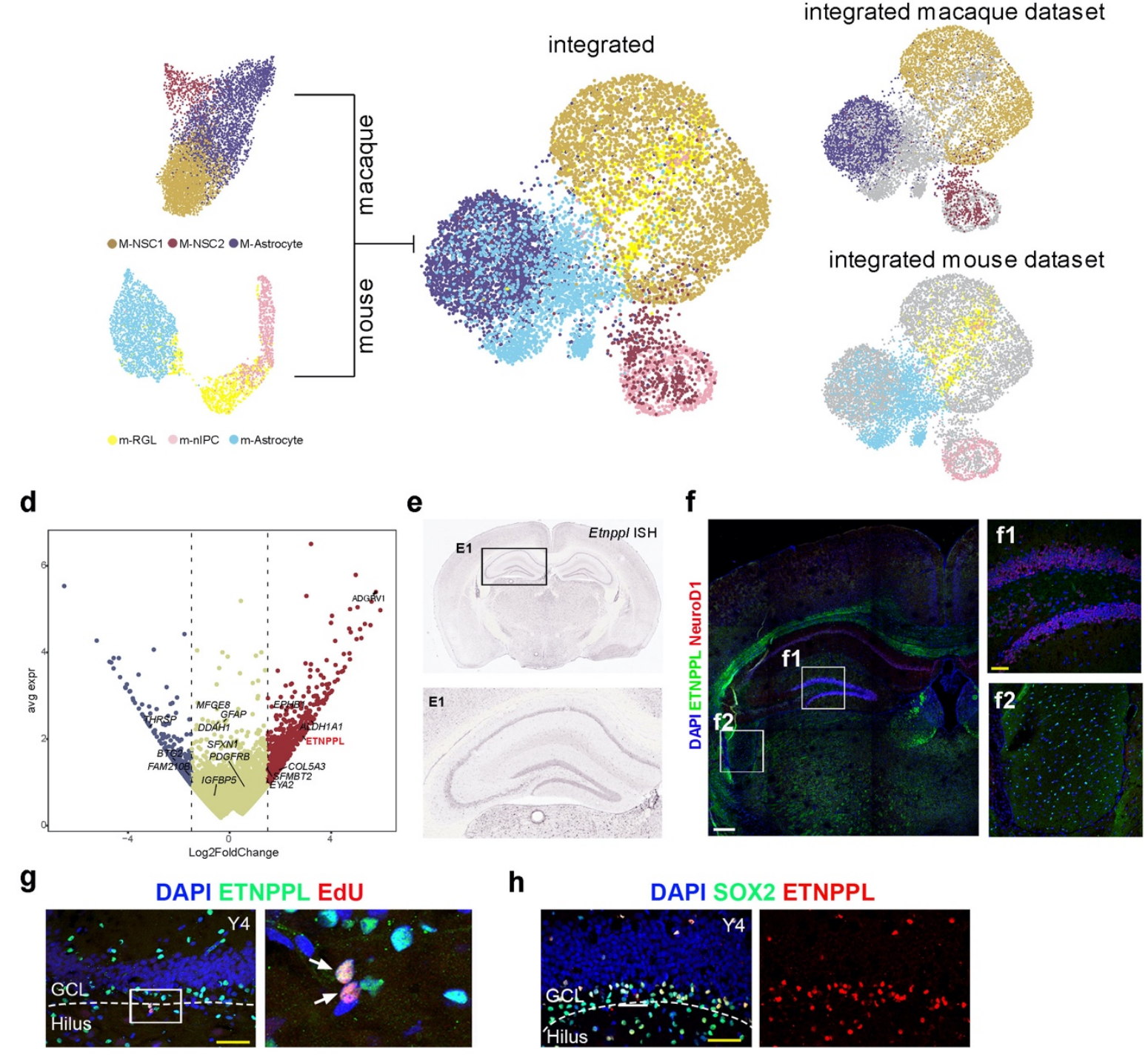

integrated macaque dataset

693

694 Extended Data Fig. 4 | Unique transcriptional features of macaque NSCs. a, Clustering of

695

696

697 


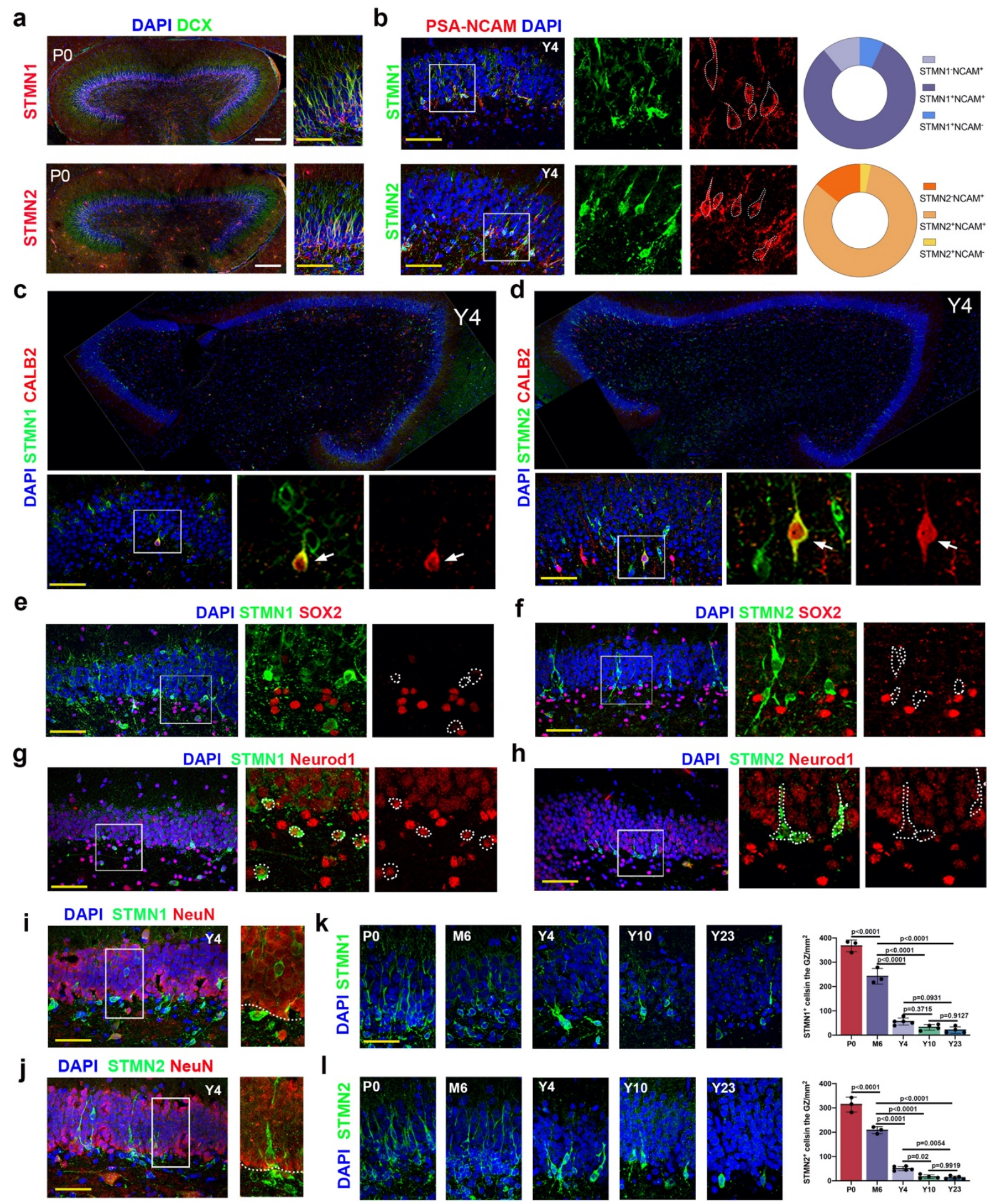

Extended Data Fig. 5 | STMN1 and STMN2 are markers for immature granule cells. a,

707 Expression of STMN1 (upper panel) and STMN2 (lower panel) with DCX in the DG of the

708 hippocampus in the P0 macaque brains. b, Colocalization analysis of STMN1 (upper panel) and STMN2 (lower panel) with PSA-NCAM showing that more than $80 \%$ of cells were double 
positive. $\mathbf{c}, \mathbf{d}$, Colocalization analysis of STMN1 (c) and STMN2 (d) with CALB2; the arrows

711 indicate colocalization. e, f, Colocalization analysis of STMN1 (e) and STMN2 (f) with SOX2.

712 The dashed circles show SOX2-negative signals. $\mathbf{g}, \mathbf{h}$, Colocalization analysis of STMN1 (g)

713 and STMN2 (h) with NeuroD1. The dashed circles show NeuroD1-negative signals. i, j, 714 Colocalization analysis of STMN1 (i) and STMN2 (j) with NeuN. k, l, Time-course analysis 715 of STMN1 and STMN2 expression in the DG of the hippocampus indicating decreased but 716 persistent neurogenesis in the aged brains. Sidak's multiple comparisons test was used, each 717 dot represents a single experiment. mean \pm s.d. Scale bar in white: $500 \mu \mathrm{m}$, scale bar in yellow: $71850 \mu \mathrm{m}$. 
a

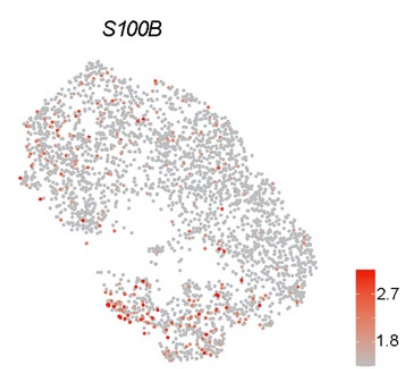

SPOCK3

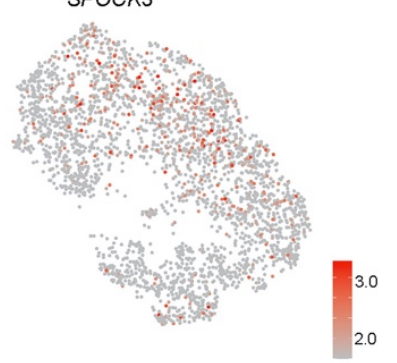

PTN

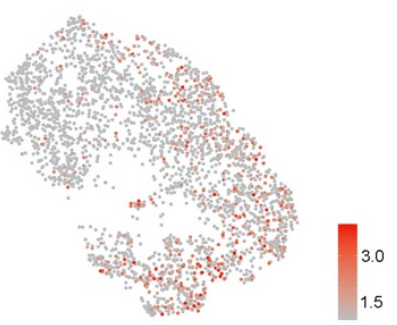

IL33

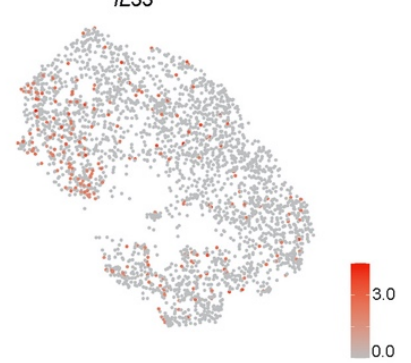

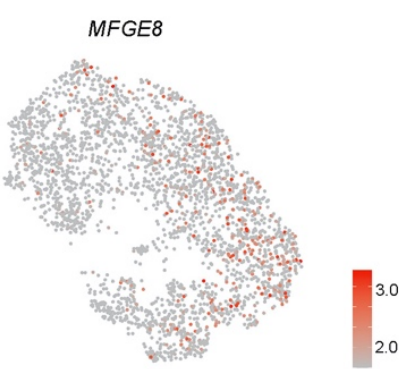

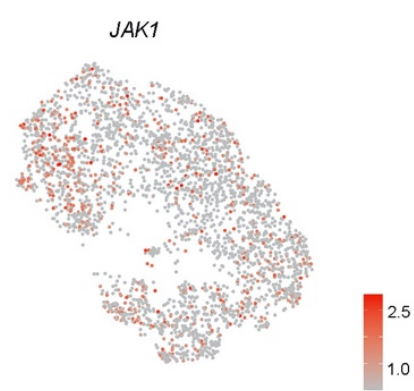

720

721 Extended Data Fig. 6 | Diversity of macaque astrocytes. a, UMAP showing the expression 722 of astrocyte subtype-specific genes.

723 
a

Microglia regional differentially expressed genes

DG CA

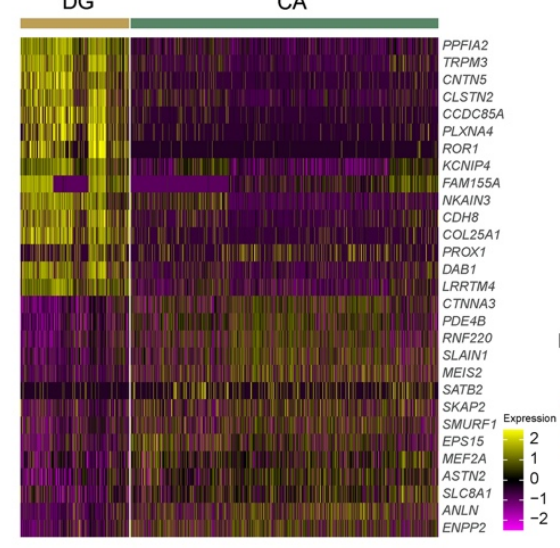

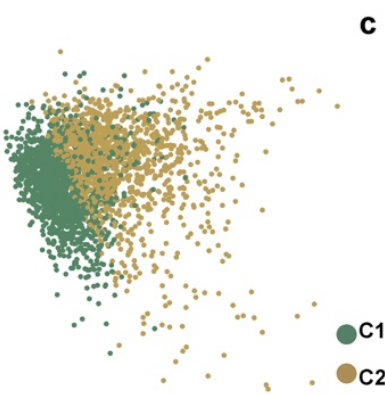

DG DEGs enrichment

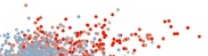

CA DEGs enrichment

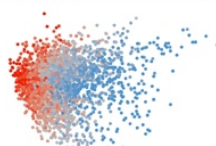

$\|_{1.5}^{15}$

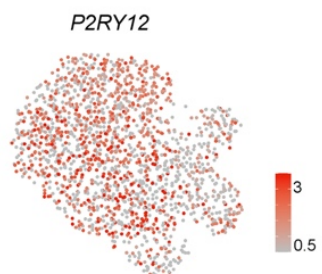

CD74

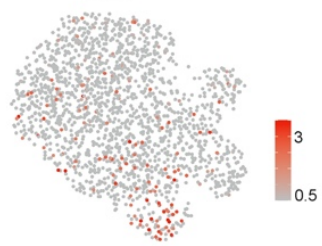

VCAN
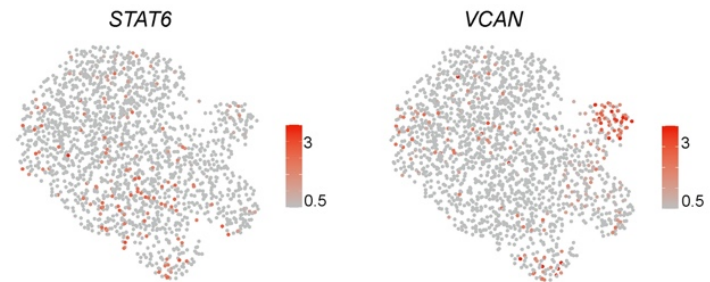

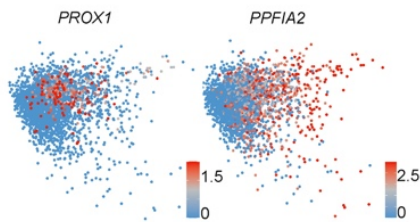

SKAP2

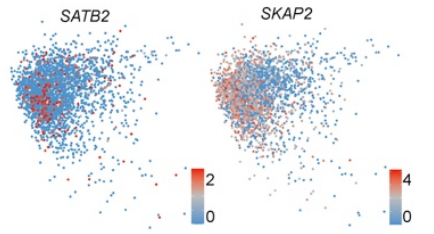

1.s

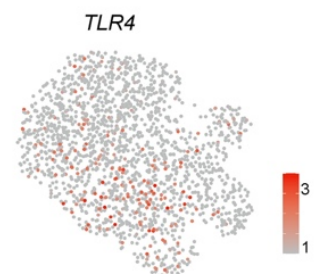

CD163

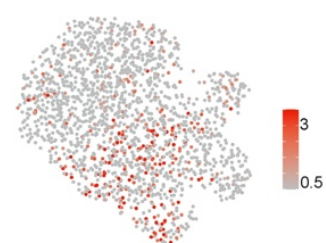

TNC

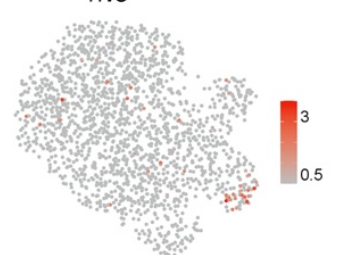

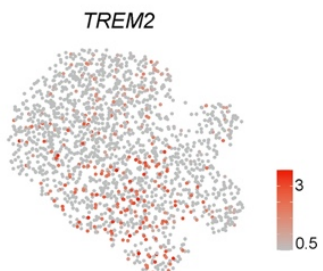

NFKB1

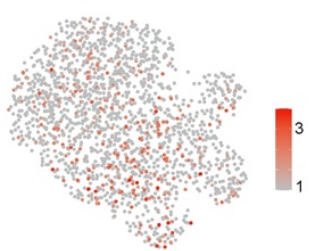

GL/3

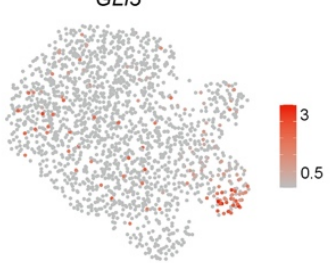

725 Extended Data Fig. 7 | Regional transcriptional profiles of microglia in the macaque

hippocampus. a, Heatmap illustrating differentially expressed genes between microglial cells from the CA and DG. b, Clustering of microglia from whole hippocampi into two groups via PCA reduction (upper panel). Visualization of the enrichment score of regional differentially expressed genes from CA and DG via a PCA plot (lower panel). c, PCA plot showing the expression of DG-enriched genes (PROX1 and PPFIA2) and CA-enriched genes (SATB2 and SKAP2). d, UMAP showing the expression of microglial subtype-specific genes. 
a

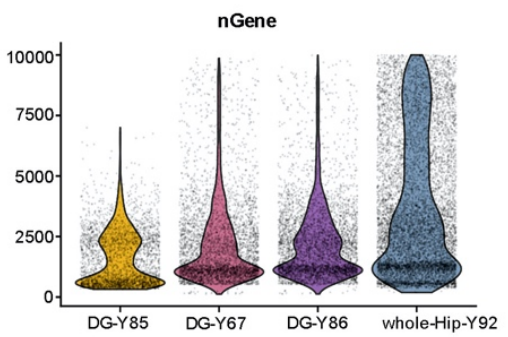

\section{b}
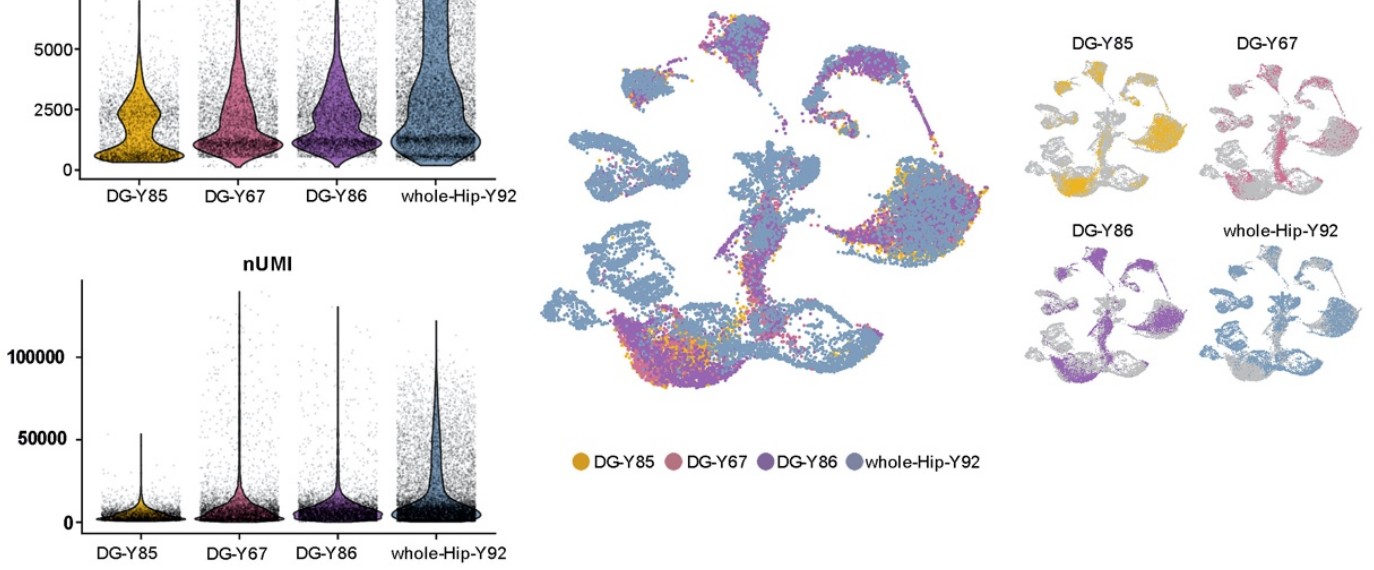

C
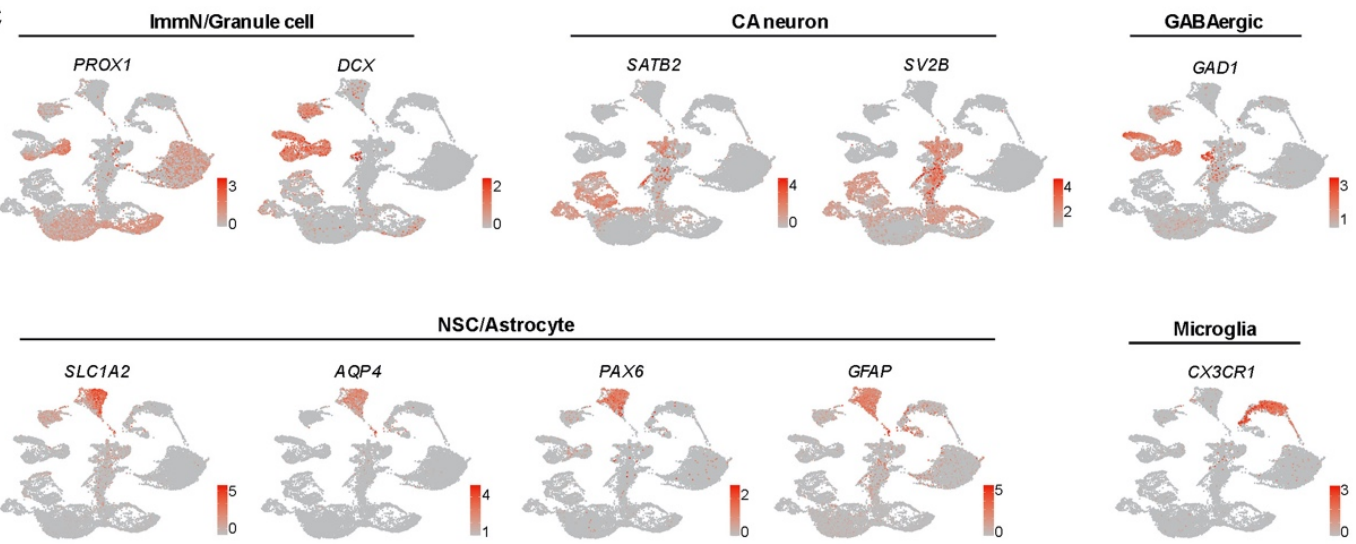

NSC/Astrocyte
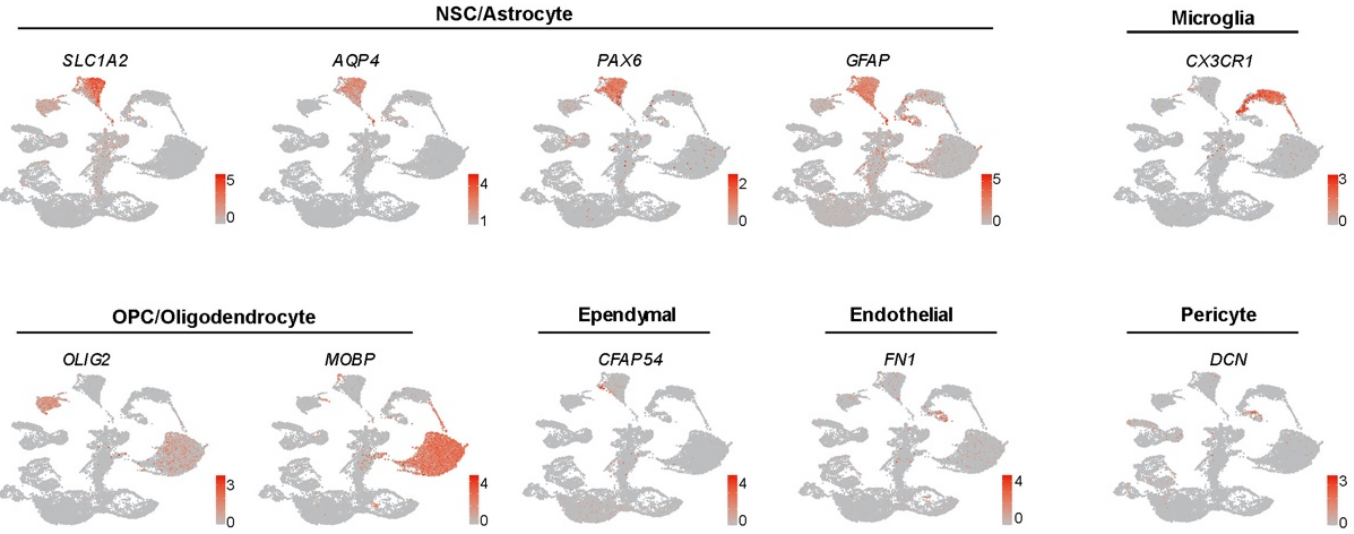

734 Extended Data Fig. 8 |Transcriptional profiles of human hippocampi. a, Quality control

735 metrics were the number of genes (nGene) and number of unique molecular identifiers (nUMI)

736 for human hippocampi samples; each dot represents a single cell. b, UMAP showing the

737 distribution of four obtained human hippocampal samples. c, The expression of well-known

738 markers for major cell types was visualized using UMAP. 
a

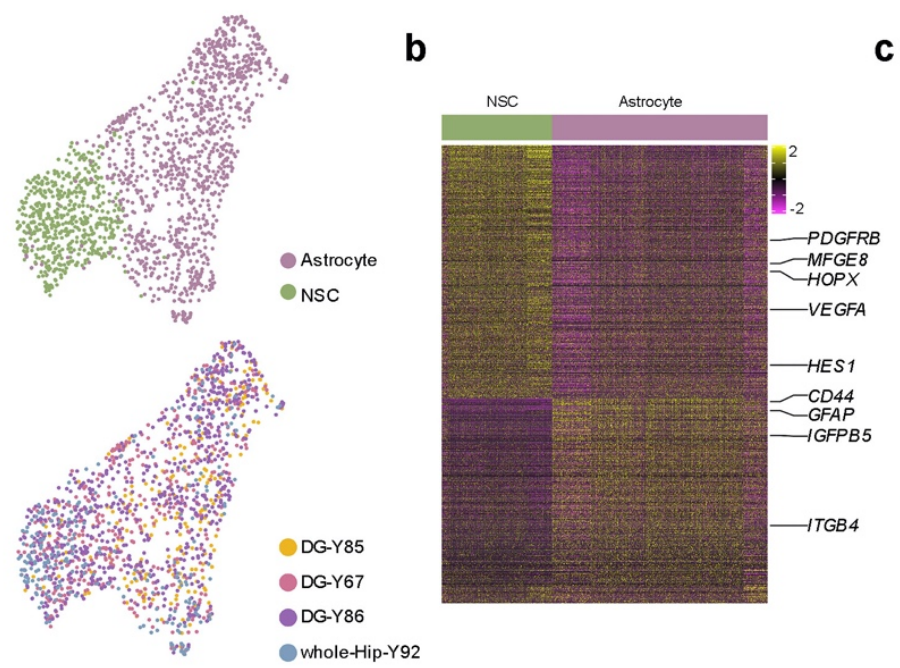

d

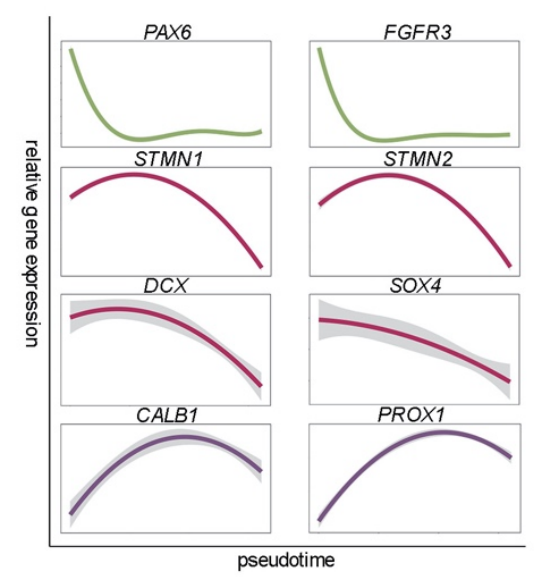

$\mathbf{f}$

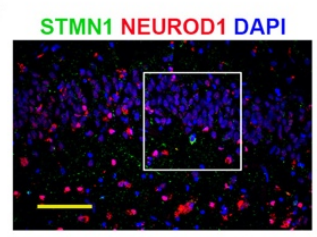

h STMN1 CALB2 DAPI
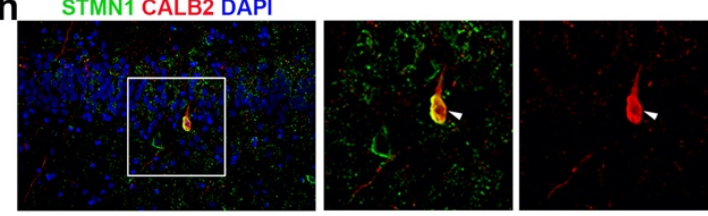

e

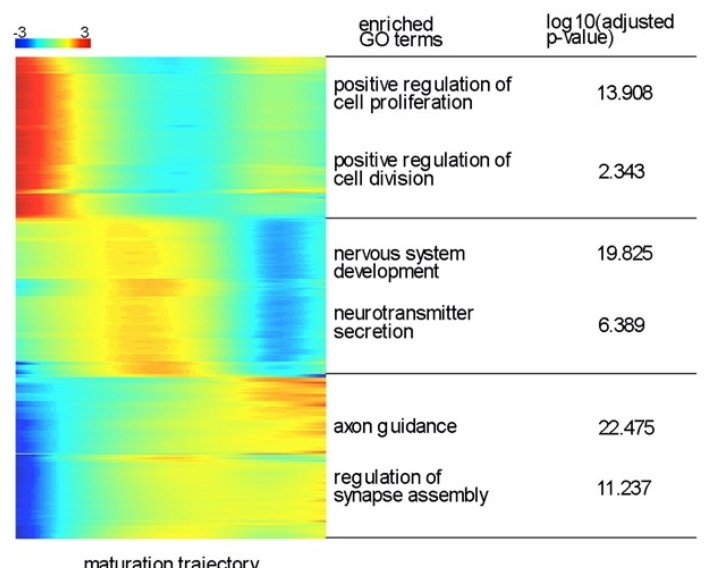

maturation trajectory
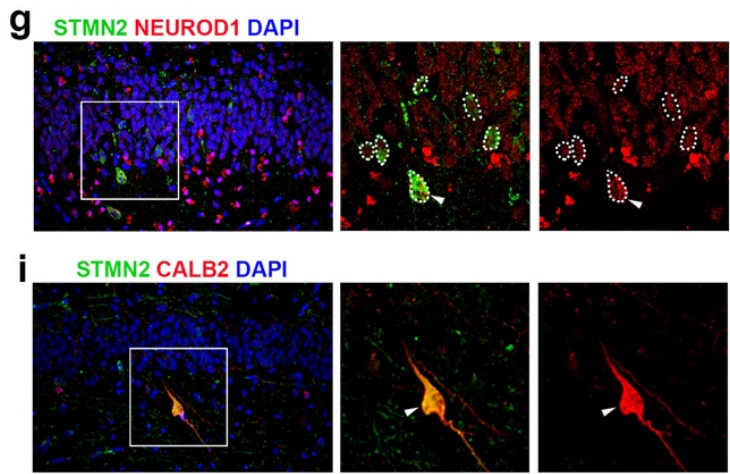

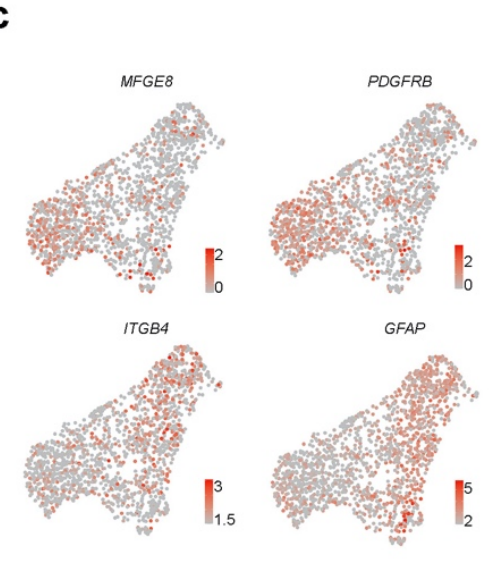

Extended Data Fig. 9 | Gene expression profiles along the human neurogenic trajectory.

743 a, UMAP showing the clustering of human NSCs and astrocytes (upper panel) and the distribution of samples (lower panel). b, Heatmap illustrating the differentially expressed genes

745 between human NSCs and astrocytes. c, UMAP showing the expression of NSC (MFGE8 and $746 P D G F R B)$ and astrocyte (ITGB4 and GFAP) enriched genes. d, Fitted curve showing the expression of representative genes along the human adult neurogenic trajectory. e, Heatmap 
748 illustrating the expression of genes that covary across pseudotime (left panel). The enriched 749 Gene Ontology terms are also shown (right panel). f, g, Both STMN1 and STMN2 were 750 expressed in human NEROUD1-positive cells. h, i, STMN1 and STMN2 are colabeled with 751 CALB2 in the SGZ of the aged human hippocampus. Scale bar in yellow: $50 \mu \mathrm{m}$. 

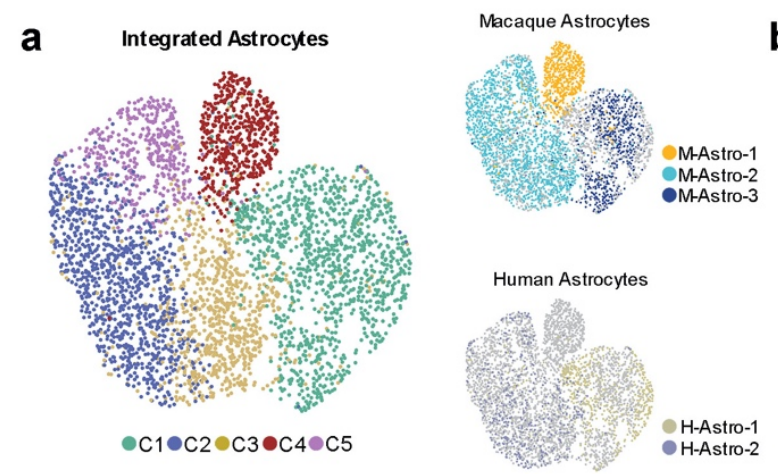

C

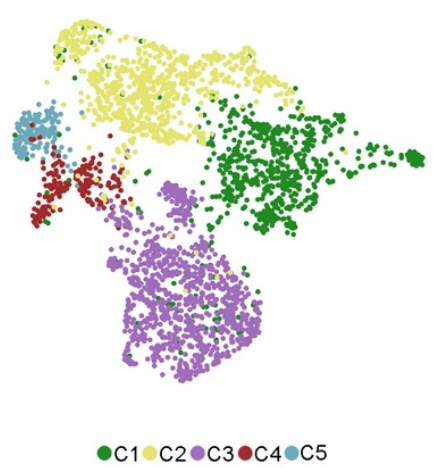

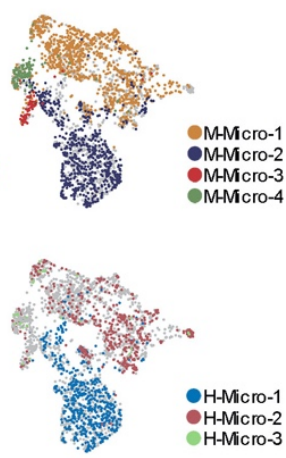

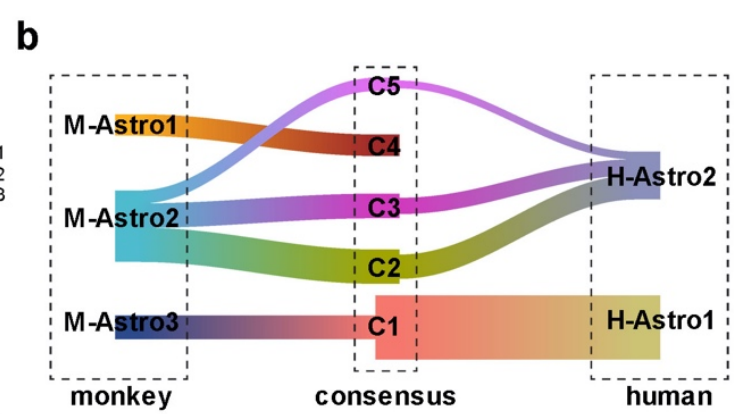

d

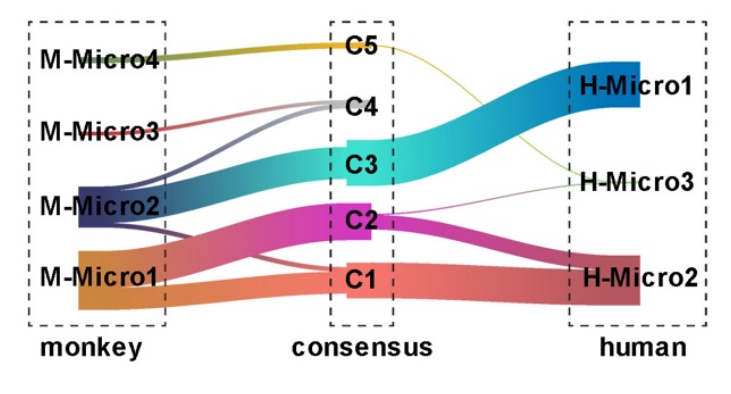

752

753

754

755

756

757

758

759

760

\section{Extended Data Fig. 10 | Comparison of astrocyte and microglia subtypes from macaques} and humans. a, The integration of macaques and human astrocytes was visualized using UMAP. b, River plot illustrating the correspondence between macaque and human astrocyte subtypes. c, The integration of macaques and human microglia is visualized using UMAP. d, River plot illustrating the correspondence between macaque and human microglial subtypes. 


\section{Supplementary Table1.}

Summary of sample information for macaques and humans.

\section{Supplementary Table2.}

Differentially expressed genes among major cell types identified in macaque hippocampi.

\section{Supplementary Table3.}

Differentially expressed genes between Astrocytes from CA and DG.

\section{Supplementary Table4.}

Differentially expressed genes between Astrocyte, NSC1 and NSC2.

\section{Supplementary Table5.}

Differentially expressed genes between macaque and mouse NSC.

\section{Supplementary Table6.}

Differentially expressed genes among major cell types identified in human hippocampi.

\section{Supplementary Table7.}

Differentially expressed genes between human NSC and Astrocytes.

\section{Supplementary Table8.}

Detailed information of Antibodies used in the study 


\section{Supplementary Files}

This is a list of supplementary files associated with this preprint. Click to download.

- SupplementaryTable3.xlsx

- SupplementaryTable8.xlsx

- SupplementaryTable1.xlsx

- SupplementaryTable7.xlsx

- SupplementaryTable6.xlsx

- SupplementaryTable5.xlsx

- SupplementaryTable4.xlsx

- SupplementaryTable2.xlsx 